\title{
Concept design of low frequency telescope for CMB B-mode polarization satellite LiteBIRD
}

\author{
Sekimoto, Y., Ade, P. A., Adler, A., Allys, E., Arnold, K., et \\ al.
}

Y. Sekimoto, P. A. R. Ade, A. Adler, E. Allys, K. Arnold, D. Auguste, J. Aumont, R. Aurlien, J. Austermann, C. Baccigalupi, A. J. Banday, R. Banerji, R. B. Barreiro, S. Basak, J. Beall, D. Beck, S. Beckman, J. Bermejo, P. de Bernardis, M. Bersanelli, J. Bonis, J. Borrill, F. Boulanger, S. Bounissou, M. Brilenkov, M. Brown, M. Bucher, E. Calabrese, P. Campeti, A. Carones, F. J. Casas, A. Challinor, V. Boulanger, S. Bounissou, M. Brilenkov, M. Brown, M. Bucher, E. Calabrese, P. Campeti, A. Carones, F. J. Casas, A. Challinor, Chan, K. Cheung, Y. Chinone, J. F. Cliche, L. Colombo, F. Columbro, J. Cubas, A. Cukierman, D. Curtis, G. D'Alessandro, N.
Dachlythra, M. De Petris, C. Dickinson, P. Diego-Palazuelos, M. Dobbs, T. Dotani, L. Duband, S. Duff, J. M. Duval, K. Ebisawa, T. Elleflot, H. K. Eriksen, J. Errard, T. Essinger-Hileman, F. Finelli, R. Flauger, C. Franceschet, U. Fuskeland, M. Galloway, K. Ganga, J. R. Gao, R. Genova-Santos, M. Gerbino, M. Gervasi, T. Ghigna, E. Gjerløw, M. L. Gradziel, J. Grain, F. Grupp, A. Gruppuso, J. E. Gudmundsson, T. de Haan, N. W. Halverson, P. Hargrave, T. Hasebe, M. Hasegawa, M. Hattori, M. Hazumi, S. Henrot-Versillé, D. Herman, D. Herranz, C. A. Hill, G. Hilton, Y. Hirota, E. Hivon, R. A. Hlozek, Y. Hoshino, E. de la Hoz, J. Hubmayr, K. Ichiki, T. iida, H. Imada, K. Ishimura, H. Ishino, G. Jaehnig, T. Kaga, S. Kashima, N. Katayama, A. Kato, T. Kawasaki, R. Keskitalo, T. Kisner, Y. Kobayashi, N. Kogiso, A. Kogut, K. Kohri, E. Komatsu, K. Komatsu, K. Konishi, N. Krachmalnicoff, I. Kreykenbohm, C. L. Kuo, A Kushino, L. Lamagna, J. V. Lanen, M. Lattanzi, A. T. Lee, C. Leloup, F. Levrier, E. Linder, T. Louis, G. Luzzi, T. Maciaszek, B. Maffei, D. Maino, M. Maki, S. Mandelli, E. Martinez-Gonzalez, S. Masi, T. Matsumura, A. Mennella, M. Migliaccio, Y. Minanmi, K. Mitsuda, J. Montgomery, L. Montier, G. Morgante, B. Mot, Y. Murata, J. A. Murphy, M. Nagai, Y. Nagano, T. Nagasaki, R. Nagata, S. Nakamura, T. Namikawa, P. Natoli, S. Nerval, T. Nishibori, H. Nishino, C. O'Sullivan, H. Ogawa, H. Ogawa, S. Oguri, H. Ohsaki, I. S. Ohta, N. Okada, N. Okada, L. Pagano, A. Paiella, D. Paoletti, G. Patanchon, J. Peloton, F. Piacentini, G. Pisano, G. Polenta, D. Poletti, T. Prouvé, G. Puglisi, D. Rambaud, C. Raum, S. Realini, M. Reinecke, M. Remazeilles, A. Ritacco, G. Roudil, J. A. Rubino-Martin, M. Russell, H. Sakurai, Y. Sakurai, M. Sandri, M. Sasaki, G. Savini, D. Scott, J. Seibert, B. Sherwin, K. Shinozaki, M. Shiraishi, P. Shirron, G. Signorelli, G. Smecher, S. Stever, R. Stompor, H. Sugai, S. Sugiyama, A. Suzuki, J. Suzuki, T. L. Svalheim, E. Switzer, R. Takaku, H. Takakura, S Takakura, Y Takase, Y Takeda, A Tartari, E Taylor, Y Terao, H. Thommesen, K. Thompson. B. Thorne, T Toda, M Tomasi, M. Tominaga, N. Trappe, M. Tristram, M. Tsuji, M. Tsujimoto, C. Tucker, J. Ullom, G. Vermeulen, P. Vielva, F. Villa, M. Vissers, Tomasi, M. Tominaga, N. Trappe, M. Tristram, M. Tsuji, M. Tsujimoto, C. Tucker, J. Ullom, G. Vermeulen, P. Vielva, F. Villa, M. Vissers, A. Zonca, "Concept design of low frequency telescope for CMB B-mode polarization satellite LiteBIRD," Proc. SPIE 11453, Millimeter, Submillimeter, and Far-Infrared Detectors and Instrumentation for Astronomy X, 1145310 (16 December 2020); doi: $10.1117 / 12.256184$

Event: SPIE Astronomical Telescopes + Instrumentation, 2020, Online Only 


\section{Concept Design of Low Frequency Telescope for CMB B-mode Polarization satellite LiteBIRD}

Y. Sekimoto ${ }^{1,2,3}$, P.A.R. Ade ${ }^{4}$, A. Adler ${ }^{5}$, E. Allys ${ }^{6}$, K. Arnold ${ }^{7}$, D. Auguste ${ }^{8}$, J. Aumont ${ }^{9}$, R. Aurlien ${ }^{10}$, J. Austermann ${ }^{11}$, C. Baccigalupi ${ }^{12}$, A.J. Banday ${ }^{9}$, R. Banerji ${ }^{10}$, R.B. Barreiro ${ }^{13}$,

S. Basak ${ }^{14}$, J. Beall ${ }^{11}$, D. Beck ${ }^{15}$, S. Beckman ${ }^{16}$, J. Bermejo ${ }^{17}$, P. de Bernardis ${ }^{18}$,

M. Bersanelli ${ }^{19}$, J. Bonis ${ }^{8}$, J. Borrill ${ }^{20,21}$, F. Boulanger ${ }^{6}$, S. Bounissou ${ }^{22}$, M. Brilenkov ${ }^{10}$,

M. Brown ${ }^{23}$, M. Bucher ${ }^{24}$, E. Calabrese ${ }^{4}$, P. Campeti ${ }^{12}$, A. Carones ${ }^{25}$, F.J. Casas ${ }^{13}$,

A. Challinor ${ }^{26,27,28}$, V. Chann ${ }^{29}$, K. Cheung ${ }^{16}$, Y. Chinone ${ }^{30}$, J.F. Cliche ${ }^{31}$, L. Colombo ${ }^{19}$,

F. Columbro ${ }^{18}$, J. Cubas $^{32}$, A. Cukierman ${ }^{16,15}$, D. Curtis ${ }^{21}$, G. D'Alessandro ${ }^{18}$,

N. Dachlythra ${ }^{33}$, M. De Petris ${ }^{18}$, C. Dickinson ${ }^{23}$, P. Diego-Palazuelos ${ }^{13}$, M. Dobbs ${ }^{31}$, T. Dotani ${ }^{1}$, L. Duband ${ }^{34}$, S. Duff ${ }^{11}$, J.M. Duval ${ }^{34}$, K. Ebisawa ${ }^{1}$, T. Elleflot ${ }^{35}$, H.K. Eriksen ${ }^{10}$, J. Errard ${ }^{24}$, T. Essinger-Hileman ${ }^{36}$, F. Finelli ${ }^{37}$, R. Flauger ${ }^{7}$, C. Franceschet ${ }^{19}$, U. Fuskeland ${ }^{10}$, M. Galloway ${ }^{10}$, K. Ganga ${ }^{24}$, J.R. Gao $^{38}$, R. Genova-Santos ${ }^{39}$, M. Gerbino ${ }^{40}$, M. Gervasi ${ }^{41}$,

T. Ghigna ${ }^{42,43}$, E. Gjerløw ${ }^{10}$, M.L. Gradziel ${ }^{44}$, J. Grain ${ }^{22}$, F. Grupp ${ }^{45}$, A. Gruppuso ${ }^{37}$, J.E. Gudmundsson ${ }^{33}$, T. de Haan $^{3}$, N.W. Halverson ${ }^{46}$, P. Hargrave ${ }^{4}$, T. Hasebe ${ }^{1}$, M. Hasegawa ${ }^{3}$, M. Hattori ${ }^{47}$, M. Hazumi ${ }^{3,1,43,48}$, S. Henrot-Versillé ${ }^{8}$, D. Herman ${ }^{10}$, D. Herranz ${ }^{13}$, C.A. Hill ${ }^{35,16}$, G. Hilton ${ }^{11}$, Y. Hirota ${ }^{49}$, E. Hivon ${ }^{50}$, R.A. Hlozek ${ }^{29}$, Y. Hoshino ${ }^{51}$, E. de la Hoz ${ }^{13}$, J. Hubmayr ${ }^{11}$, K. Ichiki ${ }^{52}$, T. Iida ${ }^{53}$, H. Imada $^{43,54}$, K. Ishimura ${ }^{55}$, H. Ishino ${ }^{56}$, G. Jaehnig ${ }^{46}$, T. Kaga ${ }^{1}$, S. Kashima ${ }^{54}$, N. Katayama ${ }^{43}$, A. Kato ${ }^{3,48}$, T. Kawasaki ${ }^{57}$,

R. Keskitalo ${ }^{20,21}$, T. Kisner ${ }^{20,21}$, Y. Kobayashi ${ }^{49}$, N. Kogiso ${ }^{58}$, A. Kogut ${ }^{36}$, K. Kohri ${ }^{3}$,

E. Komatsu ${ }^{59}$, K. Komatsu ${ }^{56}$, K. Konishi ${ }^{49}$, N. Krachmalnicoff ${ }^{12}$, I. Kreykenbohm ${ }^{60}$,

C.L. Kuo ${ }^{61,15}$, A. Kushino ${ }^{62}$, L. Lamagna ${ }^{18}$, J.V. Lanen ${ }^{11}$, M. Lattanzi ${ }^{63}$, A.T. Lee ${ }^{35,16}$, C. Leloup ${ }^{24}$, F. Levrier ${ }^{6}$, E. Linder ${ }^{35,21}$, T. Louis ${ }^{8}$, G. Luzzi ${ }^{64}$, T. Maciaszek ${ }^{65}$, B. Maffei ${ }^{22}$, D. Maino ${ }^{19}$, M. Maki ${ }^{3}$, S. Mandellii ${ }^{19}$, E. Martinez-Gonzalez ${ }^{13}$, S. Masi ${ }^{18}$, T. Matsumura ${ }^{43}$, A. Mennella ${ }^{19}$, M. Migliaccio ${ }^{25}$, Y. Minami ${ }^{3}$, K. Mitsuda ${ }^{54}$, J. Montgomery ${ }^{31}$, L. Montier ${ }^{9}$,

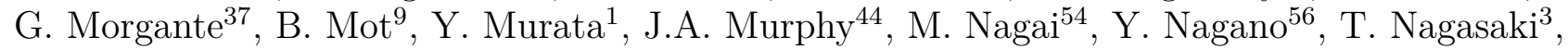

R. Nagata ${ }^{1}$, S. Nakamura ${ }^{66}$, T. Namikawa ${ }^{26}$, P. Natoli $^{40}$, S. Nerval ${ }^{29}$, T. Nishibori ${ }^{67}$,

H. Nishino ${ }^{30}$, C. O'Sullivan ${ }^{68}$, H. Ogawa ${ }^{58}$, H. Ogawa ${ }^{1}$, S. Oguri ${ }^{1}$, H. Ohsaki ${ }^{49}$, I.S. Ohta ${ }^{69}$, N. Okada ${ }^{1}$, N. Okada ${ }^{58}$, L. Pagano ${ }^{40}$, A. Paiella ${ }^{18}$, D. Paoletti ${ }^{37}$, G. Patanchon ${ }^{24}$, J. Peloton ${ }^{8}$,

F. Piacentini ${ }^{18}$, G. Pisano ${ }^{18,4}$, G. Polenta ${ }^{70}$, D. Poletti ${ }^{12}$, T. Prouvé ${ }^{34}$, G. Puglisi ${ }^{15}$,

D. Rambaud $^{9}$, C. Raum $^{16}$, S. Realini ${ }^{19}$, M. Reinecke ${ }^{59}$, M. Remazeilles ${ }^{23}$, A. Ritacco $^{22,6}$,

G. Roudil ${ }^{9}$, J.A. Rubino-Martin ${ }^{39}$, M. Russell ${ }^{7}$, H. Sakurai ${ }^{71}$, Y. Sakurai ${ }^{43}$, M. Sandri ${ }^{37}$, M. Sasaki ${ }^{60}$, G. Savini ${ }^{72}$, D. Scott ${ }^{73}$, J. Seibert ${ }^{7}$, B. Sherwin ${ }^{26,28,35}$, K. Shinozaki ${ }^{67}$, M. Shiraishi ${ }^{74}$, P. Shirron ${ }^{36}$, G. Signorelli ${ }^{75}$, G. Smecher ${ }^{76}$, S. Stever ${ }^{56,43}$, R. Stompor ${ }^{24}$,

H. Sugai ${ }^{43}$, S. Sugiyama ${ }^{51}$, A. Suzuki ${ }^{35}$, J. Suzuki ${ }^{3}$, T.L. Svalheim ${ }^{10}$, E. Switzer ${ }^{36}$, R. Takaku ${ }^{1,77}$, H. Takakura ${ }^{2,1}$, S. Takakura ${ }^{43}$, Y. Takase ${ }^{56}$, Y. Takeda ${ }^{1}$, A. Tartari ${ }^{75}$, E. Taylor ${ }^{16}$, Y. Terao ${ }^{49}$, H. Thommesen ${ }^{10}$, K.L. Thompson ${ }^{61,15}$, B. Thorne ${ }^{42}$, T. Toda ${ }^{56}$,

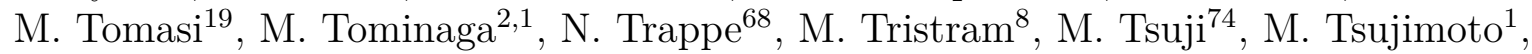
C. Tucker ${ }^{4}$, J. Ullom ${ }^{11}$, G. Vermeulen ${ }^{78}$, P. Vielva ${ }^{13}$, F. Villa ${ }^{37}$, M. Vissers ${ }^{11}$, N. Vittorio ${ }^{25}$, I. Wehus ${ }^{10}$, J. Weller ${ }^{59}$, B. Westbrook ${ }^{16}$, J. Wilms ${ }^{60}$, B. Winter ${ }^{72,79}$, E.J. Wollack ${ }^{36}$, N.Y. Yamasaki ${ }^{1}$, T. Yoshida ${ }^{1}$, J. Yumoto ${ }^{49}$, M. Zannoni ${ }^{41}$, and A. Zonca ${ }^{80}$

${ }^{1}$ Japan Aerospace Exploration Agency (JAXA), Institute of Space and Astronautical Science (ISAS), Sagamihara, Kanagawa 252-5210, Japan

Millimeter, Submillimeter, and Far-Infrared Detectors and Instrumentation for Astronomy X, edited by Jonas Zmuidzinas, Jian-Rong Gao, Proc. of SPIE Vol. 11453, 1145310 (c) 2020 SPIE · CCC code: $0277-786 X / 20 / \$ 21 \cdot$ doi: $10.1117 / 12.2561841$ 
${ }^{2}$ The University of Tokyo, Department of Astronomy, Tokyo 113-0033, Japan

${ }^{3}$ High Energy Accelerator Research Organization (KEK), Tsukuba, Ibaraki 305-0801, Japan

${ }^{4}$ Cardiff University, School of Physics and Astronomy, Cardiff CF10 3XQ, UK

${ }^{5}$ Stockholm University

${ }^{6}$ Laboratoire de Physique de l'École Normale Supérieure, ENS, Université PSL, CNRS, Sorbonne Université, Université de Paris, 75005 Paris, France

${ }^{7}$ University of California, San Diego, Department of Physics, San Diego, CA 92093-0424, USA

${ }^{8}$ Université Paris-Saclay, CNRS/IN2P3, IJCLab, 91405 Orsay, France

${ }^{9}$ IRAP, Université de Toulouse, CNRS, CNES, UPS, (Toulouse), France

${ }^{10}$ University of Oslo, Institute of Theoretical Astrophysics, NO-0315 Oslo, Norway

${ }^{11}$ National Institute of Standards and Technology (NIST), Boulder, Colorado 80305, USA

${ }^{12}$ International School for Advanced Studies (SISSA), Via Bonomea 265, 34136, Trieste, Italy

${ }^{13}$ Instituto de Fisica de Cantabria (IFCA, CSIC-UC), Avenida los Castros SN, 39005, Santander, Spain

${ }^{14}$ School of Physics, Indian Institute of Science Education and Research Thiruvananthapuram, Maruthamala PO,

Vithura, Thiruvananthapuram 695551, Kerala, India

${ }^{15}$ Stanford University, Department of Physics, CA 94305-4060, USA

${ }^{16}$ University of California, Berkeley, Department of Physics, Berkeley, CA 94720, USA

${ }^{17}$ Instituto Universitario de Microgravedad Ignacio Da Riva (IDR/UPM), Plaza Cardenal Cisneros 3, 28040 - Madrid, Spain

${ }^{18}$ Dipartimento di Fisica, Università La Sapienza, P. le A. Moro 2, Roma, Italy and INFN Roma

${ }^{19}$ Dipartimento di Fisica, Università degli Studi di Milano, INAF-IASF Milano, and Sezione INFN Milano

${ }^{20}$ Lawrence Berkeley National Laboratory (LBNL), Computational Cosmology Center, Berkeley, CA 94720, USA

${ }^{21}$ University of California, Berkeley, Space Science Laboratory, Berkeley, CA 94720, USA

${ }^{22}$ Institut d'Astrophysique Spatiale (IAS), CNRS, UMR 8617, Université Paris-Sud 11, Bâtiment 121, 91405 Orsay, France

${ }^{23}$ University of Manchester, Manchester M13 9PL, United Kingdom

${ }^{24}$ AstroParticle and Cosmology (APC) - University Paris Diderot, CNRS/IN2P3, CEA/Irfu, Obs de Paris, Sorbonne Paris Cité, France

${ }^{25}$ Dipartimento di Fisica, Università di Roma "Tor Vergata", and Sezione INFN Roma2

${ }^{26}$ DAMTP, Centre for Mathematical Sciences, Wilberforce Road, Cambridge CB3 0WA, U.K.

${ }^{27}$ Institute of Astronomy, Madingley Road, Cambridge CB3 0HA, U.K.

${ }^{28}$ Kavli Institute for Cosmology Cambridge, Madingley Road, Cambridge CB3 0HA, U.K.

${ }^{29}$ University of Toronto

${ }^{30}$ University of Tokyo, School of Science, Research Center for the Early Universe, RESCEU

${ }^{31}$ McGill University, Physics Department, Montreal, QC H3A 0G4, Canada

${ }^{32}$ Universidad Politécnica de Madrid

${ }^{33}$ Stockholm University

${ }^{34}$ Univ. Grenoble Alpes, CEA, IRIG-DSBT, 38000 Grenoble, France

${ }^{35}$ Lawrence Berkeley National Laboratory (LBNL), Physics Division, Berkeley, CA 94720, USA

${ }^{36}$ NASA Goddard Space Flight Center

${ }^{37}$ INAF - OAS Bologna, via Piero Gobetti, 93/3, 40129 Bologna (Italy)

${ }^{38}$ SRON Netherlands Institute for Space Research

${ }^{39}$ Instituto de Astrofisica de Canarias (IAC), Spain

${ }^{40}$ Dipartimento di Fisica e Scienze della Terra, Università di Ferrara and Sezione INFN di Ferrara, Via Saragat 1, 44122 Ferrara, Italy

${ }^{41}$ University of Milano Bicocca, Physics Department, p.zza della Scienza, 3, 20126 Milan Italy

${ }^{42}$ University of Oxford

${ }^{43}$ Kavli Institute for the Physics and Mathematics of the Universe (Kavli IPMU, WPI), UTIAS, The University of

Tokyo, Kashiwa, Chiba 277-8583, Japan

${ }^{44}$ National University of Ireland Maynooth

${ }^{45} \mathrm{MPE}$

${ }^{46}$ Center for Astrophysics and Space Astronomy, University of Colorado, Boulder, CO, 80309, USA

${ }^{47}$ Tohoku University, Graduate School of Science, Astronomical Institute, Sendai, 980-8578, Japan

${ }^{48}$ The Graduate University for Advanced Studies (SOKENDAI), Miura District, Kanagawa 240-0115, Hayama, Japan

${ }^{49}$ The University of Tokyo, Tokyo 113-0033, Japan

${ }^{50}$ Institut d'Astrophysique de Paris, CNRS/Sorbonne Université, Paris France

${ }^{51}$ Saitama University, Saitama 338-8570, Japan 


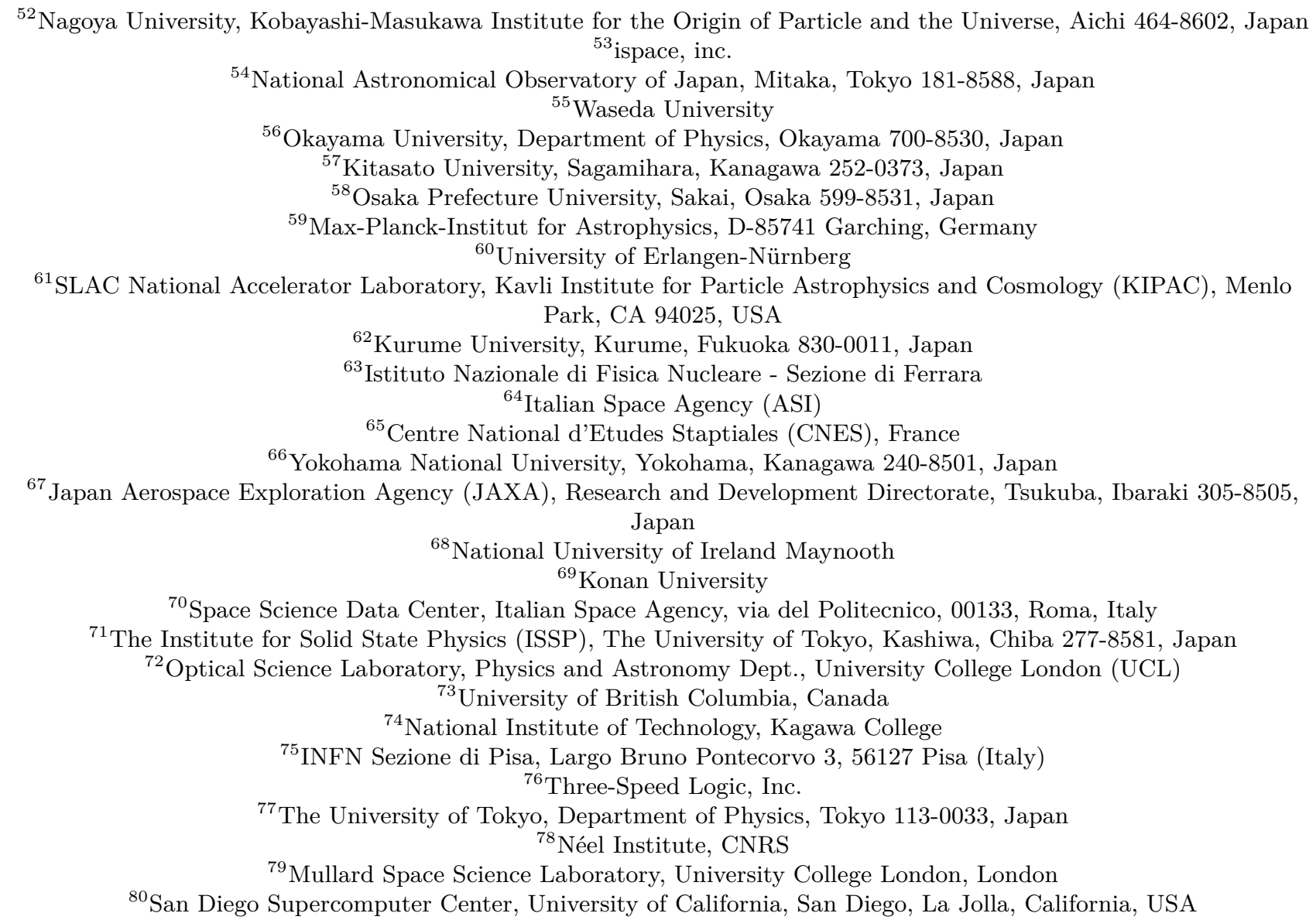

\begin{abstract}
LiteBIRD has been selected as JAXA's strategic large mission in the 2020s, to observe the cosmic microwave background (CMB) B-mode polarization over the full sky at large angular scales. The challenges of LiteBIRD are the wide field-of-view (FoV) and broadband capabilities of millimeter-wave polarization measurements, which are derived from the system requirements. The possible paths of stray light increase with a wider FoV and the far sidelobe knowledge of $-56 \mathrm{~dB}$ is a challenging optical requirement. A crossed-Dragone configuration was chosen for the low frequency telescope (LFT : 34-161 GHz), one of LiteBIRD's onboard telescopes. It has a wide field-of-view $\left(18^{\circ} \times 9^{\circ}\right)$ with an aperture of $400 \mathrm{~mm}$ in diameter, corresponding to an angular resolution of about 30 arcminutes around $100 \mathrm{GHz}$. The focal ratio $\mathrm{f} / 3.0$ and the crossing angle of the optical axes of $90^{\circ}$ are chosen after an extensive study of the stray light. The primary and secondary reflectors have rectangular shapes with serrations to reduce the diffraction pattern from the edges of the mirrors. The reflectors and structure are made of aluminum to proportionally contract from warm down to the operating temperature at $5 \mathrm{~K}$. A $1 / 4$ scaled model of the LFT has been developed to validate the wide field-of-view design and to demonstrate the reduced far sidelobes. A polarization modulation unit (PMU), realized with a half-wave plate (HWP) is placed in front of the aperture stop, the entrance pupil of this system. A large focal plane with approximately 1000 AlMn TES detectors and frequency multiplexing SQUID amplifiers is cooled to $100 \mathrm{mK}$. The lens and sinuous antennas have broadband capability. Performance specifications of the LFT and an outline of the proposed verification plan are presented.
\end{abstract}

Keywords: Cosmic microwave background, space program, millimeter-wave polarization, cryogenic telescope

Send correspondence to Y. Sekimoto, E-mail: sekimoto.yutaro@jaxa.jp 


\section{INTRODUCTION}

LiteBIRD, the Lite (Light) satellite for the study of $B$-mode polarization and Inflation from cosmic background Radiation Detection, observes the cosmic microwave background (CMB) polarization over the full sky at large angular scales. ${ }^{1-3}$ Cosmological inflation predicts primordial gravitational waves, which imprinted large-scale curl ( $B$-mode) patterns on the CMB polarization map. ${ }^{4-7}$ Measurements of the CMB $B$-mode signals are known as the best probe to detect the primordial gravitational waves and to measure the inflation energy. The scientific objective of LiteBIRD is to test major inflationary models. ${ }^{8}$ The power of the $B$-modes is proportional to the tensor-to-scalar ratio, $r$. The current upper limit on $r$ is $r<0.044 .{ }^{9}$ The mission goal of LiteBIRD is to measure $r$ with a precision of $\delta r<0.001$, which provides a crucial test of cosmic inflation. The required angular coverage is $2<\ell<200$, where $\ell$ is the multipole moment.

LiteBIRD has been selected as JAXA's strategic large mission in the late 2020s. It will be launched with an $\mathrm{H} 3$ vehicle for three years of observations at the Lagrangian point (L2) of the Earth-Sun system. It is a spinning satellite with a precession angle $(\alpha)$ of $45^{\circ}$ and spin angle $(\beta)$ of $50^{\circ}$ with spin rate of $0.05 \mathrm{rpm}$ and precession period of 180 minutes, which are optimized from crossing angles and revisits of previously scanned regions. The concept design has been studied by researchers from Japan, U.S., Canada, and Europe since September 2016.

LiteBIRD observes millimeter waves from $34 \mathrm{GHz}$ to $448 \mathrm{GHz}$ with two instruments, LFT and MHFT. ${ }^{10,11}$ Both instruments have the same relative bandwidth of min: max frequencies $=1: 5$. LFT will explore synchrotron and CMB emission, while MHFT covers CMB emission and will also extend to higher frequencies to explore the dust contribution. The bands in common between the two telescopes, i.e. $89-161 \mathrm{GHz}$, allow reduction of systematics associated with the telescopes, and add redundancy. A transmissive half-wave plate (HWP) for polarization modulation has a limited bandwidth, and so LiteBIRD has two instruments to cover the frequency bands. Both instruments are operated at cryogenic temperature of $5 \mathrm{~K}$ to reduce the photon noise. The focal plane design is based on multi-chroic TES detectors at $100 \mathrm{mK}$ operation. ${ }^{12,13}$ Cryogenic chain of LiteBIRD is described by Hasebe et al. ${ }^{14}$ and Duval et al. ${ }^{15}$

Challenges for LiteBIRD are wide field-of-view (FoV) and broadband capabilities of millimeter-wave polarization measurements, which are derived from the sensitivity specifications. The wide FoV corresponds to a large focal plane area; a detector pixel has different spill-over or edge-taper depending on the pixel position on the focal plane. The possible paths of stray light increase with a wider FoV. A stable system is also required to perform the all sky survey.

LiteBIRD is currently under the conceptual study phase. It is important to define preliminary design specifications in order to make progress on the system design. The derivation of the detailed requirements and the detailed design study are moving in parallel, and affect each other iteratively. In this paper we introduce a list of design specifications in this phase. Based on further simulation-based studies of the error budget allocation over the entire system, the numbers we list for the design specifications may change.

\section{OVERVIEW OF LFT}

LFT has been designed to meet specifications described in the next section. This section describes a brief overview of LFT before describing design details. LFT is a wide field-of-view telescope designed to observe the $\mathrm{CMB}$ and synchrotron radiation in the frequencies of $34-161 \mathrm{GHz}$, as shown in Figure 1. The aperture diameter is $400 \mathrm{~mm}$. The angular resolution is $24-71$ arcminutes. LFT is operated at cryogenic temperature of $5 \mathrm{~K}$ to reduce the optical loading and is surrounded by radiators called V-grooves. The thermal design of LiteBIRD is described in Hasebe et al. ${ }^{14}$ LFT has a crossed Dragone antenna made of aluminum. A frame structure at $5 \mathrm{~K}$ supports all components: the PMU (polarization modulation unit); focal plane; primary and secondary reflectors; and absorbers. An earlier design ${ }^{2}$ has been updated.

A PMU with a transmissive HWP (half-wave plate) ${ }^{16}$ is mounted in front of the aperture stop. LFT focal plane is based on multi-chroic TES detectors at $100 \mathrm{mK}$ operation. ${ }^{12,13}$ There are interfaces with the LFT PMU and the LFT focal plane.

\section{LFT DESIGN SPECIFICATIONS}

The performance specifications for LFT are as follows. 


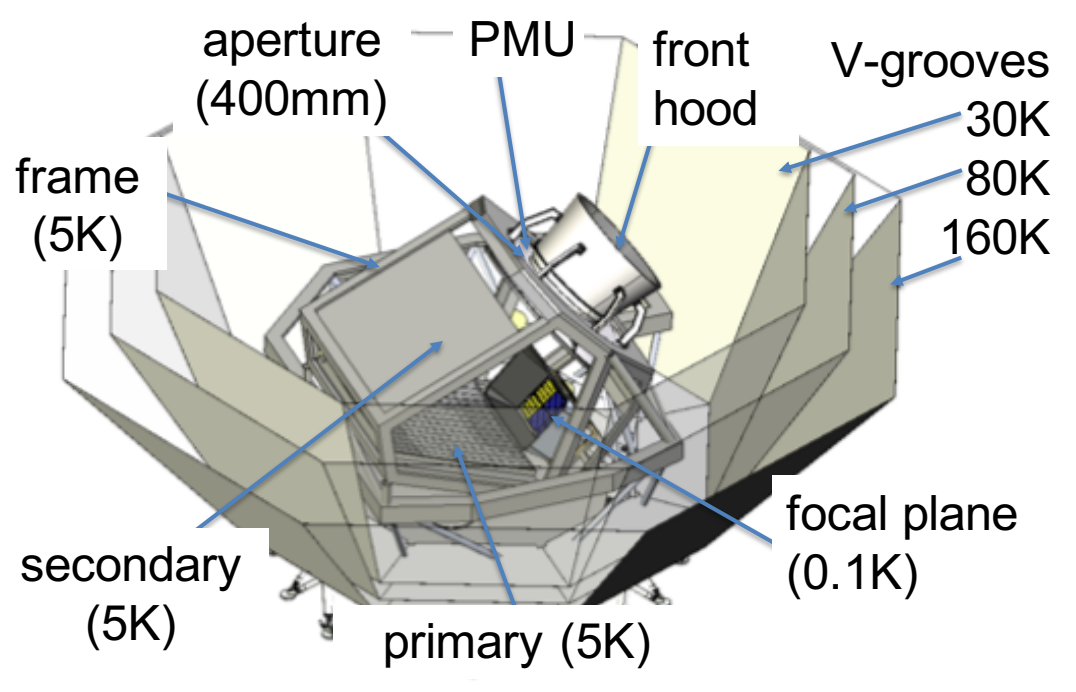

Figure 1. Overview of low frequency telescope (LFT). MHFT and side panels are not shown for clarity.

\subsection{Frequency bands and noise}

\section{Frequency coverage $34-161 \mathrm{GHz}$}

Band sensitivities LFT shall have the array sensitivities as tabulated in Table 1, which shall satisfy the maplevel sensitivity specifications. The sensitivity is limited by the number of pixels, which is closely related with the field of view of the telescope. The noise of the detector in a pixel is limited by the optical loading.

Table 1. Performance specifications of LFT. The bandwidth (BW) is (High - Low)/Center frequency.

\begin{tabular}{|c|c|c|c|c|c|c|}
\hline $\begin{array}{r}\text { Center freq. } \\
\mathrm{GHz}\end{array}$ & BW & $\begin{array}{c}\text { Beam fwhm } \\
\text { [arcmin] }\end{array}$ & $\begin{array}{l}\text { pixel dia. } \\
{[\mathrm{mm}]}\end{array}$ & No. det & $\begin{array}{c}\mathrm{NET}_{\text {array }} \\
{[\mu \mathrm{Krts}]}\end{array}$ & $\begin{array}{l}\text { Pol. sensitivity } \\
{[\mu \mathrm{K} \text { arcmin }]}\end{array}$ \\
\hline 40 & 0.30 & 70.5 & 32 & 48 & 18.5 & 37.4 \\
\hline 50 & 0.30 & 58.5 & 32 & 24 & 16.5 & 33.5 \\
\hline 60 & $\overline{0.23}$ & 51.1 & 32 & 48 & 10.5 & 21.3 \\
\hline 68 & 0.23 & 41.6 & 16 & 144 & 9.8 & \multirow[b]{2}{*}{16.9} \\
\hline 68 & 0.23 & 47.1 & 32 & 24 & 15.7 & \\
\hline 78 & 0.23 & 36.9 & 16 & 144 & 7.7 & \multirow{2}{*}{12.1} \\
\hline 78 & 0.23 & 43.8 & 32 & 48 & 9.5 & \\
\hline 89 & 0.23 & 33.0 & 16 & 144 & 6.1 & \multirow{2}{*}{11.3} \\
\hline 89 & 0.23 & 41.5 & 32 & 24 & 14.2 & \\
\hline 100 & 0.23 & 30.2 & 16 & 144 & 5.1 & 6.6 \\
\hline 119 & 0.30 & 26.3 & 16 & 144 & 3.8 & 4.6 \\
\hline 140 & 0.30 & 23.7 & 16 & 144 & 3.6 & 4.8 \\
\hline
\end{tabular}

Band shape The frequency bandpasses are defined by a combination of superconducting band-pass filters on the wafer, ${ }^{12}$ and the use of quasi-optical metal-mesh filters ${ }^{17}$ in front of the focal plane to reject higher frequencies. Lower frequencies than the defined band (red-leak) might contribute to sidelobes due to the distorted beam pattern. The red-leak is rejected only by a superconducting band-pass filter on the wafer. ${ }^{12}$ Higher frequencies than the defined band (blue-leak) might contribute to noise due to far-infrared radiation. The blue leak is rejected by both the on-chip filter and the quasi-optical metal mesh filter in front of the focal plane.

1/f noise The knee frequency of the post-demodulation $1 / f$ noise should be below $0.1 \mathrm{mHz}$ (assuming a $0.05 \mathrm{rpm}$ 
spin rate, precession angle $\alpha=45^{\circ}$ and spin angle $\beta=50^{\circ}$ ). The knee frequency of the raw $1 / f$ noise should be well below $3.1 \mathrm{~Hz}(46 \mathrm{rpm} \times 4)$. The $46 \mathrm{rpm}$ value is the LFT HWP rotation rate. In the HWP failure mode, the pair-differenced $f_{\text {knee }}$ is $20 \mathrm{mHz}$ for individual detectors and $100, \mathrm{mHz}$ for the common mode.

Data loss and operational duty cycle The operating life of the instruments should be long enough to perform observations for 3 years. The system shall have an operational duty cycle of $85 \%$ for science observations, including all downtime for cryogenic cycling, detector operation preparation, and data transfer. Data loss due to cosmic ray glitches should be less than $5 \%$.

\subsection{Beam}

Angular resolution The angular resolution of each detector response should be sufficient to cover the required angular scales of $2<\ell<200$, where $\ell$ is the spherical harmonic index. It shall have a FWHM of $80^{\prime}$ or better. Angular resolution should be better than $30^{\prime}$ at $100 \mathrm{GHz}$, for measuring the recombination bump, which is the prominent structure at degree scales in the $B$-mode power spectrum coming from the primordial gravitational waves. It shall also be better than $80^{\prime}$ at $40 \mathrm{GHz}$, for dealing with point sources.

Pointing offset knowledge The pointing offset knowledge should be less than $2.1^{\prime}{ }^{18,19}$

Far sidelobe knowledge The extended component of the far sidelobe should be known at a precision level of $-56 \mathrm{~dB} \cdot{ }^{20,21}$ Radiation from the Galactic plane through the far sidelobes contaminates the signal and therefore the inferred power spectrum. The far sidelobe is currently defined as the domain located above $0.2 \mathrm{rad}$

Small scale feature of sidelobe The small-scale features of the far sidelobes should be known at a precision level of $-33 \mathrm{~dB}$, more specifically defined by the following equation: (intensity $/ 0.05 \%) \times\left(\text { diameter } / 30^{\prime}\right)^{2}$, where the diameter is the FWHM of the small-scale features due to possible optical ghosts or optical multiple reflections.

Near sidelobe knowledge The beam pattern of near sidelobes (out to $10^{\circ}$ from the co-polar beam peak) should be known at a precision level of $-30 \mathrm{~dB}$. Also, it should be confirmed to be consistent with its designed pattern at a precision level of $10 \%$ or better.

Beam stability knowledge The beam-shape stability over time, should be better than $0.46 \%$ (synchronous) $/ 2 \%$ ( random) for beam width, and better than $1.7^{\prime \prime}$ (synchronous) / $16^{\prime \prime}$ (random) for pointing, better than $0.086 \%$ (synchronous) / $2.7 \%$ (random) at the third flattening (often called ellipticity), better than $-46 \mathrm{~dB}$ at sidelobes around several to 30 degrees. ${ }^{19}$ The time scale of the synchronous beam fluctuation is $163 \mathrm{msec}$ for LFT in which HWP rotates by $45^{\circ}$, while "random" is a component that fluctuates randomly over time. They correspond to "differential beam shape" and are also related to optical qualities of the instrument in the broad sense. Note that in the case of a perfect polarization modulator, differential beam effects are negligibly small. Therefore beam stability specifications are tied to imperfections of the polarization modulation system.

\subsection{Polarization}

Knowledge of polarization efficiency The polarization efficiency knowledge should be better than $0.2 \%$.

Absolute polarization angle knowledge (monopole) The absolute polarization angle knowledge on the stable monopole component should be better than $2.7^{\prime} .^{18,19}$

Polarization modulation The modulation frequency should be $>4 \times 0.76 \mathrm{~Hz}$, which assures 4 modulation (at least) during beam-size excursions of $30^{\prime}$. The modulation frequency should be $<4 \times 4.5 \mathrm{~Hz}$, given by an argument about the bolometer time constant.

Modulation synchronous instrumental polarization knowledge The $4 f$ synchronous instrumental polarization knowledge should be better than $0.0063 \%$. 
Table 2. Optical specifications of LFT antenna

\begin{tabular}{lc}
\hline Aperture diameter & $400 \mathrm{~mm}$ \\
Field of view & $18^{\circ} \times 9^{\circ}$ \\
Strehl ratio & 0.95 at $161 \mathrm{GHz}$ \\
Focal plane telecentricity & $<1.0^{\circ}$ \\
Focal ratio & $2.9<\mathrm{F} / \#<3.1$ \\
PSF flattening & $<5 \%$ \\
Cross polarization & $<-30 \mathrm{~dB}$ \\
Rotation of polarization angle across FoV & $< \pm 1.5^{\circ}$ \\
\hline
\end{tabular}

\subsection{Gain}

Gain variation in time The gain variation in time for a single detector should be better than $10 \%$ assuming that the gain parameter is updated every $1200 \mathrm{sec}$ (which corresponds to a $0.05 \mathrm{rpm}$ rotation period). The effective differential gain should be smaller than $0.0069 \%$ (synchronous, i.e., $163 \mathrm{msec}$ for LFT, in which the HWP rotates by $45^{\circ}$ ) / $0.3 \%$ (random).

\subsection{Other specifications}

There are other specifications. According to the system design, ${ }^{2}$ heat dissipation of LFT is limited to $4 \mathrm{~mW}$, which includes the PMU and temperature control of the LFT optical components. The minumum eigen-frequency for LFT is assumed to be $100 \mathrm{~Hz}$ and $50 \mathrm{~Hz}$ for axial and lateral axes, respectively; however, this might be optimized by a combined design with the cryo-structure of the payload module (PLM). LFT is designed to withstand quasistatic loads of $20 \mathrm{~g}$ for the axial and lateral axes. EMC/EMI specifications have been studied with simulations. ${ }^{22}$

\section{OPTICAL DESIGN}

\subsection{Antenna design}

After trade-off studies of various optical configurations among crossed-Dragone, offset-Gregorian, ${ }^{23}$ and openDragone,${ }^{24,25}$ we concluded that the crossed-Dragone antenna is the best option for LFT because of the wide-field of view and the low cross polarization. Multiple reflections of crossed-Dragone antennas have been described earlier. ${ }^{26}$

A crossed-Dragone antenna of LFT has been designed with anamorphic aspherical surfaces ${ }^{27}$ to achieve the specifications listed in Table 2. The anamorphic aspherical surface is described with the following equation for both the primary mirror $(\mathrm{PM})$ and the secondary mirror $(\mathrm{SM}):{ }^{27}$

$$
z_{m}=\frac{C_{m, x} x_{m}^{2}+C_{m, y} y_{m}^{2}}{1+\sqrt{1-\left(1+k_{m, x}\right) C_{m, x}^{2} x_{m}^{2}-\left(1+k_{m, y}\right) C_{m, y}^{2} y_{m}^{2}}}+\sum_{i=2}^{5} A_{m, i}\left[\left(1-B_{m, i}\right) x_{m}^{2}+\left(1+B_{m, i}\right) y_{m}^{2}\right]^{i},
$$

where $m=\mathrm{PM}$, SM, $C_{m, x}$ and $C_{m, y}$ are curvatures for the $x$ and $y$ directions, $k_{m, x}$ and $k_{m, y}$ are conic constants in the x and y directions, and $A_{m, i}$ and $B_{m, i}$ are aspherical coefficients.

Table 3. Optical parameters of anamorphic aspherical surfaces. ${ }^{27}$

\begin{tabular}{l|l|l|l|l|l|l|l|l}
\hline \hline & $C_{m, x} / \mathrm{mm}^{-1}$ & $C_{m, y} / \mathrm{mm}^{-1}$ & $k_{m, x}$ & $k_{m, y}$ & $y_{m, 0} / \mathrm{mm}$ & $z_{m, 0} / \mathrm{mm}$ & $\theta_{m} / \mathrm{deg}$. \\
\hline PM & $-1.60053 \times 10^{-4}$ & $-4.71355 \times 10^{-4}$ & 15.857906 & -5.174224 & 0 & 696.344 & 0 \\
\hline SM & $4.05234 \times 10^{-4}$ & $5.04062 \times 10^{-4}$ & -4.162644 & -1.282787 & -163.771 & 346.223 & 42.45664 \\
\hline FP & & & & & 550.924 & 343.223 & 90 & \\
\hline \hline & $A_{m, 2}$ & $B_{m, 2}$ & $A_{m, 3}$ & $B_{m, 3}$ & $A_{m, 4}$ & $B_{m, 4}$ & $A_{m, 5}$ & $B_{m, 5}$ \\
\hline PS & $-5.28 \times 10^{-12}$ & $-3.31 \times 10^{-1}$ & $1.63 \times 10^{-18}$ & -0.716 & $-2.50 \times 10^{-24}$ & -0.973 & $-2.17 \times 10^{-34}$ & 0.0929 \\
\hline SM & $-3.10 \times 10^{-16}$ & 59.834 & $7.42 \times 10^{-18}$ & -0.375 & $-3.45 \times 10^{-23}$ & -1.157 & $-3.89 \times 10^{-31}$ & -0.349 \\
\hline
\end{tabular}

A ray diagram of LFT is shown in Figure 2, which has an aperture diameter of $400 \mathrm{~mm}$ and an FoV of $18^{\circ} \times$ $9^{\circ}$. The aperture diameter is derived from the requirement of the angular resolution of $80^{\prime}$ at $40 \mathrm{GHz}$. The FoV 
corresponds to the focal plane area of $420 \mathrm{~mm} \times 210 \mathrm{~mm}$, which is roughly proportional to the sensitivity. This meets the sensitivity requirement in Table 1.

Optical rays are designed to have $640 \mathrm{~mm}$ diameter at the aperture from the focal plane to keep enough edge tapers at both primary and secondary reflectors. The Strehl ratio at $161 \mathrm{GHz}$ is larger than 0.95, as shown in Figure 3. Rotation of the polarization angle for the $y$-axis polarization across the field of view is shown in Figure 3 . The rotation is estimated to $< \pm 1.5^{\circ}$ according to the ray tracing simulation with a finite resistivity. The derived optical parameters are tabulated in Table 3.

The allocated volumes of LFT and MHFT are shown in Figure 4. The field of view of LFT is maximized under the volume constraint. Crossed-Dragone antennae with $\mathrm{f} / 2.5,3.0$, and 3.5 are compared. The volume is roughly proportional to the f-number. Under the volume constraint, the smaller values are preferable, but, the stray light is larger. We chose $\mathrm{f} / 3.0$ for LFT, considering focal-plane dimensions and feed parameters.

We updated the design of a crossed-Dragone antenna reported by. ${ }^{27}$ The $\mathrm{f} / 3.0$ and the crossing angle of the optical axes of $90^{\circ}$ have been chosen after an extensive study of stray light (on the right of Figure 2).

Figure 5 shows the stray light with the crossing angles; At the crossing angle of $110^{\circ}$, the direct path from the feed to the sky is small, but there are many triple reflection paths. At $82^{\circ}$, there are large direct paths. Then the $90^{\circ}$ angle moderates for both the triple reflections and direct paths.

The detector hood and front hood whose height of $500 \mathrm{~mm}$ reduces stray light to far sidelobes as shown in Figure 2. The $y$-direction of the focal plane in the focal plane coordinate (Figure 6) is limited by multiple reflections or stray light. The $x$-direction is limited by the $5 \mathrm{~K}$ allocated area of LiteBIRD, as shown in Figure 4 .

Primary and secondary mirrors have rectangular shapes of $835 \times 795 \mathrm{~mm}$ and $872 \times 739 \mathrm{~mm}$, respectively, with serrations to reduce diffraction patterns from the edges of mirrors. The mirror sizes were reduced from the previous design ${ }^{2}$ because the $2 \mathrm{~K}$ cold aperture stop was removed due to limitations of the cooling capacity and then the length between the aperture and the main reflector was reduced. The optical design is based on feed parameters as tabulated in Table 4 .
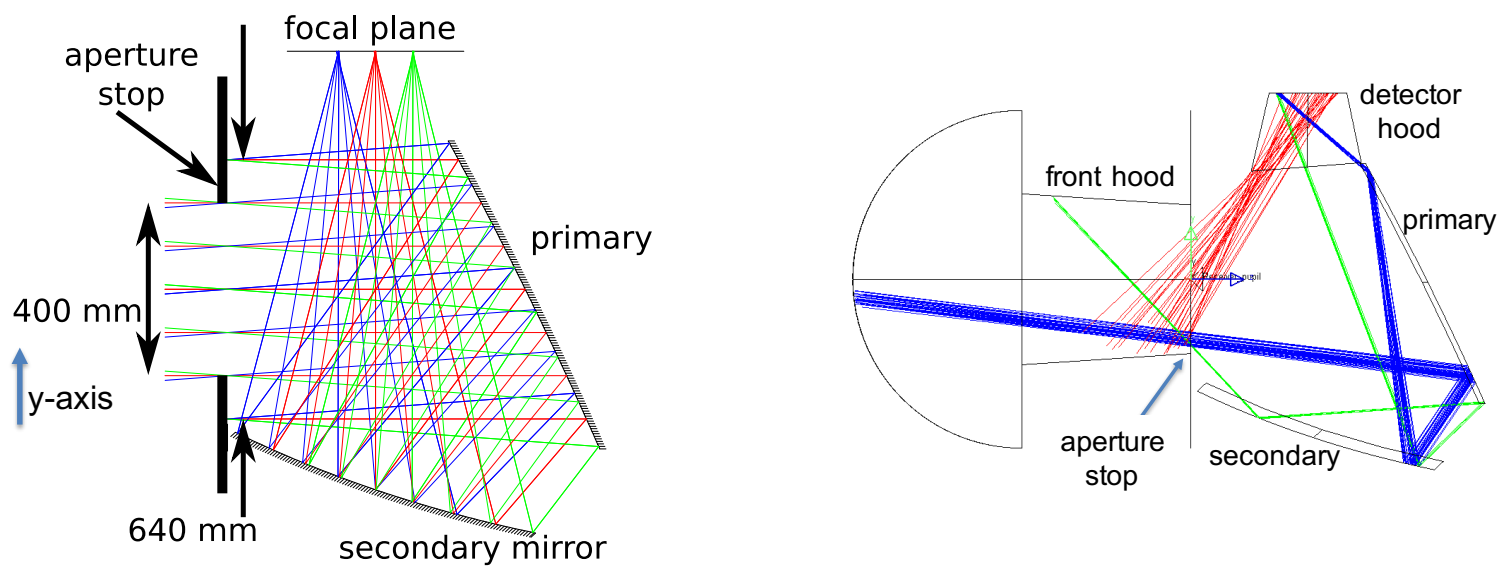

Figure 2. (Left) Ray tracing diagram of Low Frequency Telescope (LFT). Blue, Red, and Green lines show $\theta_{y}=+4.5^{\circ}$, $\theta_{y}=0^{\circ}, \theta_{y}=-4.5^{\circ}$, respectively. (Right) Possible stray light paths of LFT. Red lines show direct paths. Blue and green lines show triple reflections.

\subsection{Optical simulation}

Physical optics simulations of LFT with GRASP $10^{28}$ have been studied in the same way by Imada et al. ${ }^{29}$ Lower frequencies make it relatively difficult to meet the far sidelobe requirement due to diffraction effects. Figure 7 shows the impact of the feed sidelobes.

The LFT antenna pattern assuming a Gaussian feed is shown in the left panels of Figure 7, while the feed simulated with HFSS $^{30}$ is shown in the right ones. Upper panels show the antenna pattern of a pixel near the primary reflector, while lower ones show that of near the aperture. It is clear that the direct path from the feed 


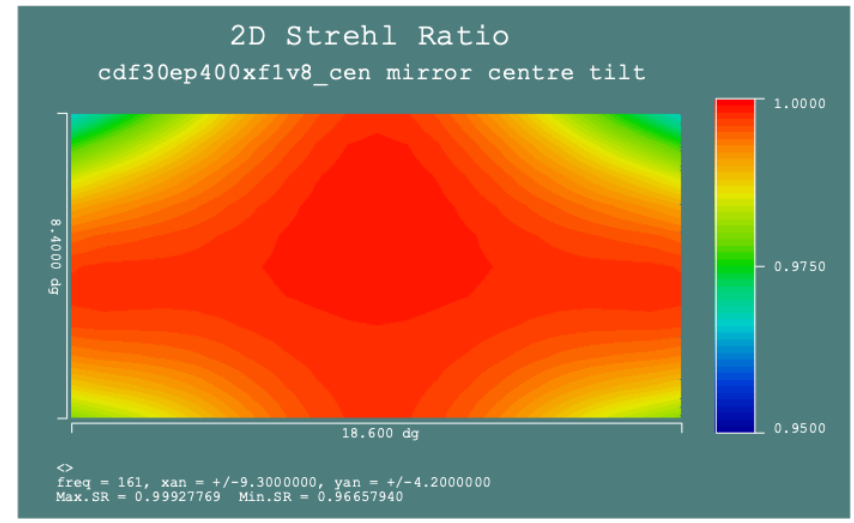

Figure 3. (Left) Map of Strehl ratio of LFT antenna at $161 \mathrm{GHz}$. (Right) Rotation of polarization angle of $y$-axis polarization across the field of view in units of degrees.

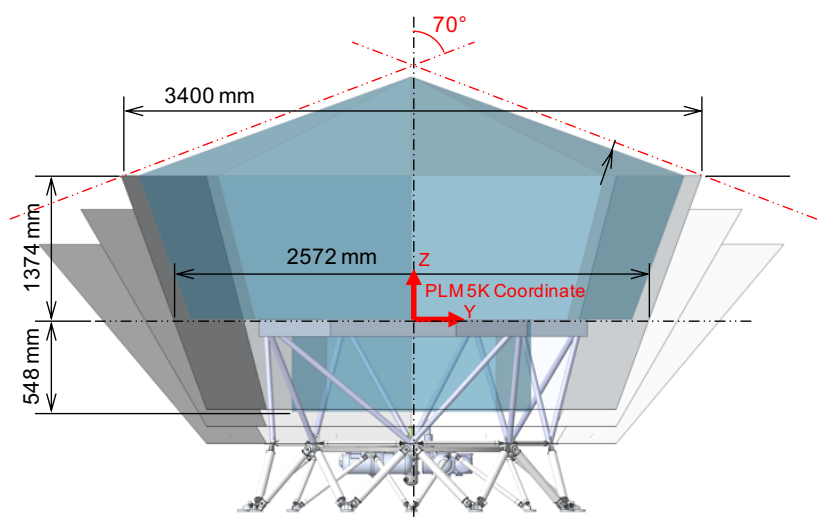

Pol Rotation Angle cdf30ep400xf1v8 w/o CAS closest spot

freq $=150\left[\mathrm{CHH}_{2}\right]$
xan $=+/-9.30000$, yan $=+/-4.2000000$
without coating

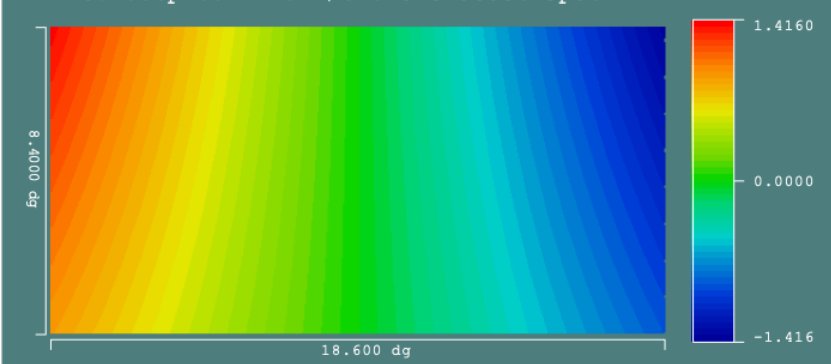

Figure 4. (Left) Usable volume of LFT and MHFT and the PLM coordinate. V-grooves are also shown. The most inner $\mathrm{V}$-groove is at $30 \mathrm{~K}$. The top of the truss is the $5 \mathrm{~K}$ structural interface for LFT and MHFT. (Right) Allocated area of LFT and MHFT and the PLM coordinate.

sidelobe contributes the far sidelobe of LFT at a level of $-60 \mathrm{~dB}$. The feed sidelobe of a pixel near the aperture contributes the point-like sidelobe due to triple reflections (feed $\rightarrow$ primary $\rightarrow$ secondary $\rightarrow$ primary $\rightarrow$ sky: shown in green). Note that there are discrepancies of the feed sidelobes at a level around $-20 \mathrm{~dB}$ between the HFSS simulation and the room-temperature measurement of the sinuous/lens feed. ${ }^{31}$

We have simulated the antenna pattern at $30 \mathrm{GHz}$, as shown in Figure 8, since a bandpass filter cannot cut off sharply at a specific frequency, e.g., $34 \mathrm{GHz}$, which causes a red leak to the sidelobe. The feed here is polarized along the $x$ axis, and located at $(x, y)=(-88 \mathrm{~mm},+44 \mathrm{~mm})$ with a diameter of $24 \mathrm{~mm}$, which is different from the current design, but the qualitative effects are the same. Several features, originating from the diffraction at the mirror edges, are shown within circles in both panels. These features are at a higher level than that of the

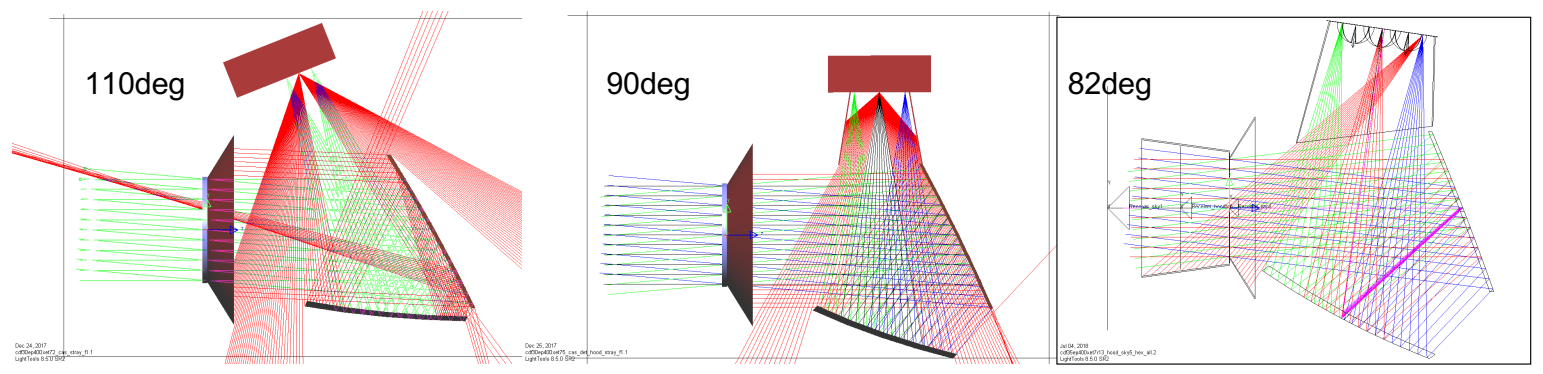

Figure 5. Stray light with the crossing angle of the optical axes of the crossed-Dragone configuration. 
Table 4. Frequency bands and feed parameters. The bandwidth (BW) is (High - Low)/Center frequency. The number (No.) of detectors is two times the number of pixels because of two orthogonal polarization detections.

\begin{tabular}{|c|r|r|r|r|r|c|c|c|}
\hline Type & $\begin{array}{r}\text { Center freq. } \\
{[\mathrm{GHz}]}\end{array}$ & BW & $\begin{array}{r}\text { Low } \\
{[\mathrm{GHz}]}\end{array}$ & $\begin{array}{r}\text { High } \\
{[\mathrm{GHz}]}\end{array}$ & $\begin{array}{r}\text { Pixel dia. } \\
{[\mathrm{mm}]}\end{array}$ & $\begin{array}{c}\text { Beam waist } \\
\text { radius }[\mathrm{mm}]\end{array}$ & No. pix & No. det. \\
\hline 1 & 40 & 0.30 & 34 & 46 & 32 & 11.64 & 24 & 48 \\
\hline & 60 & 0.23 & 53 & 67 & 32 & 11.64 & 24 & 48 \\
\hline & 78 & 0.23 & 69 & 87 & 32 & 11.64 & 24 & 48 \\
\hline 2 & 50 & 0.30 & 43 & 58 & 32 & 11.64 & 12 & 24 \\
\hline & 68 & 0.23 & 60 & 76 & 32 & 11.64 & 12 & 24 \\
\hline & 89 & 0.23 & 79 & 99 & 32 & 11.64 & 12 & 24 \\
\hline 3 & 68 & 0.23 & 60 & 76 & 16 & 5.82 & 72 & 144 \\
\hline & 89 & 0.23 & 79 & 99 & 16 & 5.82 & 72 & 144 \\
\hline 4 & 119 & 0.30 & 101 & 137 & 16 & 5.82 & 72 & 144 \\
\hline & 78 & 0.23 & 69 & 87 & 16 & 5.82 & 72 & 144 \\
\hline & 100 & 0.23 & 89 & 112 & 16 & 5.82 & 72 & 144 \\
\hline
\end{tabular}

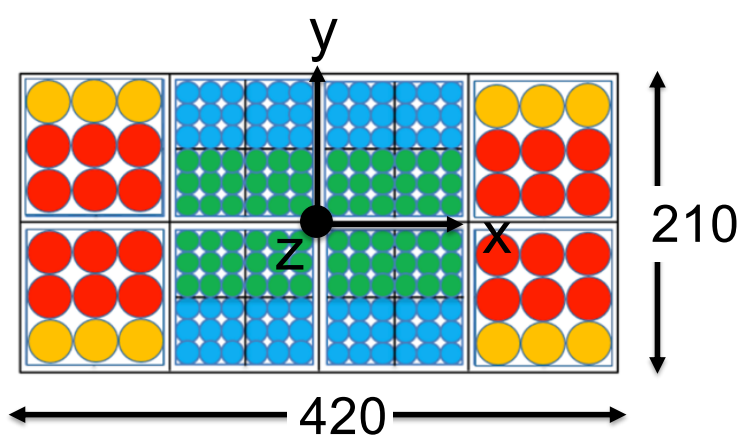

Figure 6. LFT focal plane pixel arrangement. There are eight square $(10 \mathrm{~cm} \times 10 \mathrm{~cm})$ tiles. Red, yellow, and green, blue pixels correspond Type 1, 2, 3, and 4 of Table 4, respectively. The LFT focal plane coordinate is shown in black arrows. The scales are shown in units of millimeters.

nominal diffracted point spread function (PSF).

The current simulations take into account the reflectors, the aperture stop and the front baffle with perfect absorbers. The followings items will be considered for further studies, which might generate additional side-lobes.

- Actual absorbers have finite reflections on the aperture stop, front hood, detector hood, frame, and panels. The absorbers covering the optical cavity and the focal plane are not ideal and they have frequency dependence as well as angle dependence of reflectance.

- There are multiple reflections (i.e. ghost effects) or multiple scattering among the HWP, the focal plane, the aperture stop, quasi-optical LP Filters, and the absorbers.

\subsection{Other optical components}

The aperture stop at $4.8 \mathrm{~K}$ with an inner diameter of $400 \mathrm{~mm}$ is made of millimeter absorber, TK-RAM ${ }^{32,33}$ on an aluminum plate. This works to make good beam shape for a relatively low edge taper of about $3 \mathrm{~dB}$ configuration.

Millimeter absorbers to reduce reflections are attached on the inside surface of the $5 \mathrm{~K}$ frame, which plays a role of a cavity. Eccosorb AN72 and HR10 are candidates for such absorbers; however, they have large TML (total 

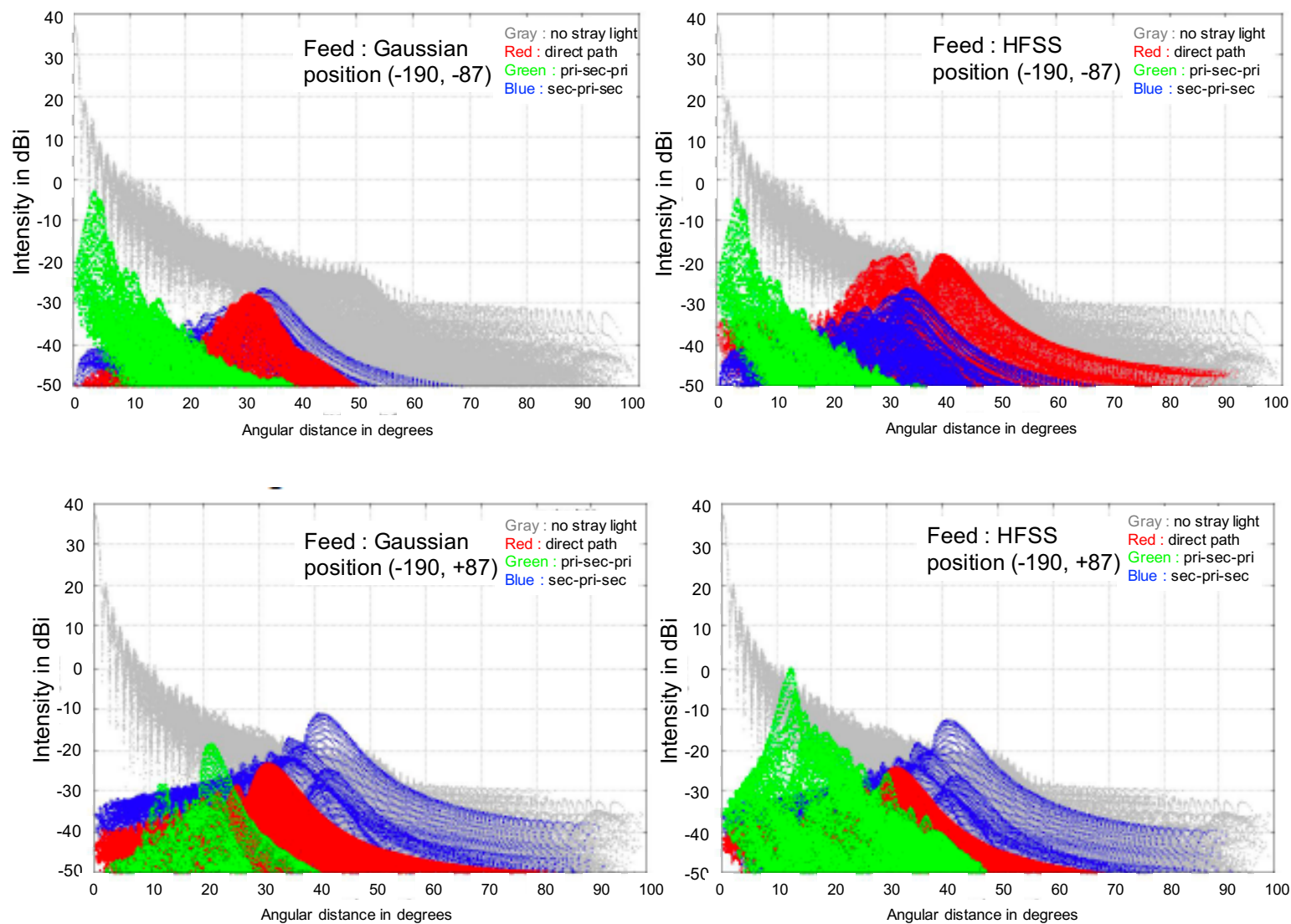

Figure 7. Optical simulation of far-field beam pattern of LFT at $34 \mathrm{GHz}$. Gray shows the nominal beam pattern without stray light, red shows the direct path from the focal plane to sky, green shows triple reflections (feed - primary secondary - primary - sky), and blue shows triple reflections (feed - secondary - primary - secondary - sky). (Top, Left) A pixel near the primary reflector around $(x, y)=(-190 \mathrm{~mm},-87 \mathrm{~mm})$ with a Gaussian feed. (Top, Right) A pixel near the primary reflector with HFSS simulation of sinuous antenna. The feed sidelobe contributes the far sidelobe of LFT due to direct path (Red). (Bottom, Left) A pixel near the aperture stop around $(x, y)=(-190 \mathrm{~mm},+87 \mathrm{~mm}) \mathrm{with}$ a Gaussian feed. (Top, Right) A pixel near the aperture stop with HFSS simulation of sinuous antenna. The feed sidelobe contributes the far sidelobe of LFT due to triple reflections (feed - primary - secondary - primary - sky: Green).

mass loss) and CVCM (collected volatile condensable materials). According to the NASA outgass database, ${ }^{34}$ AN72 washed with ethanol shows reasonable TML and CVCM.

The front-hood, as shown in Figure 9, is made of millimeter absorber Eccosorb AN72 and aluminum plate.

\subsection{Thermal control}

Temperature stability of the optical components of LFT is required to meet the specification of the single detector $f_{\text {knee }}=20 \mathrm{mHz}$, which corresponds to 50 seconds. The noise equivalent temperature (NET) of each detector is around $50 \mu \mathrm{K} / \sqrt{\mathrm{Hz}}$, so the noise is integrated to $\Delta T=7 \mu \mathrm{K}$ in the 50 seconds. It is necessary to meet the following constraint:

$$
(\Delta T)^{2} \gg \sum_{o=1}^{N_{\mathrm{o}}}\left(\delta T_{o} \times \eta_{o} \times \epsilon_{o} \times(\text { optical efficiency })\right)^{2},
$$

where $N_{\mathrm{o}}$ is the number of optical components, $\delta T_{o}$ is the temperature stability of the optical components, $\eta_{o}$ is the optical load fraction and, $\epsilon_{o}$ is the emissivity of the optical components. The optical efficiency of the feed is assumed to be 0.69 . The noise contribution of each optical component is assumed less than $2 \mu \mathrm{K}$. The derived 


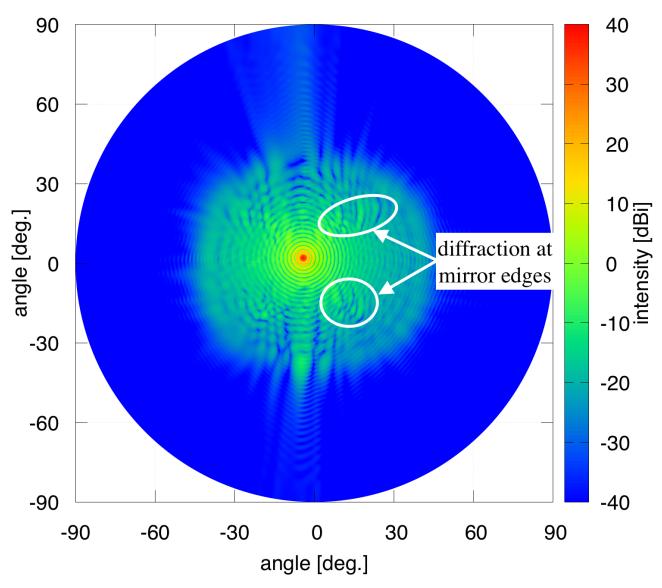

(a)

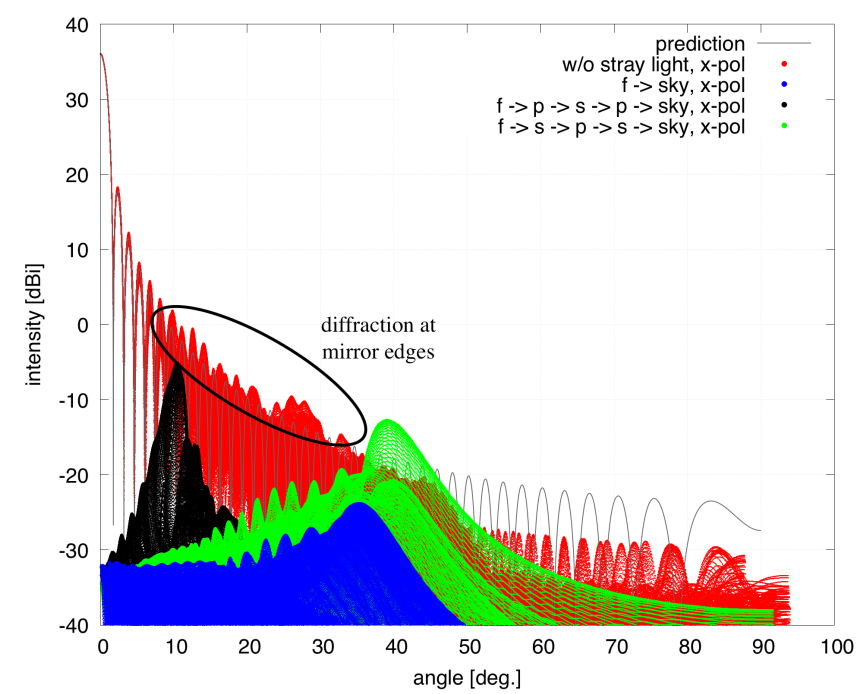

(b)

Figure 8. Physical optical simulation for $30 \mathrm{GHz}$, which is out of the band. (a) 2D map. The features from the diffraction at the mirror edges can be found at the right side of the main lobe. (b) 1D cut.

Table 5. specifications for temperature stability on the scale of 50 seconds of LFT optical components. The optical load fraction $\left(\eta_{o}\right)$ is a typical value, because it depends on the focal plane position, the feed sidelobe, and frequency. $\epsilon_{o}$ is the emissivity of the optical components.

\begin{tabular}{lccllr}
\hline Components & \multicolumn{2}{c}{ Temperature $[\mathrm{K}]$} & $\eta_{o}$ & $\epsilon_{o}$ & $\begin{array}{r}\text { Stability } \\
\text { min. }\end{array}$ \\
& 5 & 6 & 0.004 & 0.99 & {$[\mathrm{mK}]$} \\
\hline Front hood & 4.5 & 20 & 0.63 & 0.01 & 3 \\
PMU/HWP & 4.5 & 20 & 0.004 & 0.99 & 0.5 \\
PMU mount & 4.5 & 4.8 & 0.2 & 0.99 & 0.7 \\
Around aperture stop & 4.5 & 5 & 0.1 & 0.99 & 0.03 \\
5 K frame & 4.5 & 5 & 0.9 & 0.002 & 1.6 \\
LFT reflectors & 1.8 & 2 & 0.08 & 0.99 & 0.04 \\
Detector hood & 1.7 & 2 & 0.9 & 0.01 & 0.3 \\
Low-pass filter & & & & & \\
\hline
\end{tabular}

specifications on the stability of the LFT optical components are shown in Table 5. Those specifications give a rough estimate for temperature stability of $\delta T_{o} / T_{o} \sim 10^{-5}$ in the worst case, but, more accurate estimates are required, because the optical load fraction $\left(\eta_{o}\right)$ depends on the focal plane position, the feed sidelobe and the frequency, as described in section 4.2 and in Figure 7.

The temperature of the aperture stop, and other optical components, are planned to be stabilized with heaters to reduce the $1 / f$ noise level.

\section{STRUCTURE DESIGN}

The structural design of LFT is shown in Figure 9. The frame and reflectors of LFT are made of aluminum in order to shrink similarly within $0.4 \%$ from $300 \mathrm{~K}$ to $5 \mathrm{~K} .{ }^{35}$ Structural and thermal stability of the telescope is required for the all sky survey of CMB polarization observations. Aluminum has good thermal conductance at $5 \mathrm{~K}$ and is mechanically stable. The frame has structural interfaces at $5 \mathrm{~K}$ with PMU and the focal plane, which is operated at $0.1 \mathrm{~K}$. The fastener between the reflector and the frame is planned to use SUS (stainless steel) bolts. The SUS bolts generate local deformations with an area of several $\mathrm{mm}$, which does not affect on the global shape of the reflectors. The telescope is supported by trusses made of aluminum on the $5 \mathrm{~K}$ interface plate. The total mass of LFT, including the trusses, the PMU and the focal plane, is estimated to be $200 \mathrm{~kg}$. 
Table 6. Alignment specifications of LFT. All values are maxima.

\begin{tabular}{|l|l|l|l|l|}
\hline Requirement & Primary (M1) & Secondary (M2) & Frame & Combined \\
\hline Mechanical shape error & $15 \mu \mathrm{m}$ r.m.s. & $15 \mu \mathrm{m}$ r.m.s. & & $30 \mu \mathrm{m} \mathrm{r.m.s.}$ \\
\hline Alignment dx & $\pm 0.1 \mathrm{~mm}$ & $\pm 0.1 \mathrm{~mm}$ & $\pm 0.2 \mathrm{~mm}$ & $\pm 0.4 \mathrm{~mm}$ \\
\hline Alignment dy & $\pm 0.1 \mathrm{~mm}$ & $\pm 0.1 \mathrm{~mm}$ & $\pm 0.2 \mathrm{~mm}$ & $\pm 0.4 \mathrm{~mm}$ \\
\hline Alignment dz & $\pm 0.2 \mathrm{~mm}$ & $\pm 0.2 \mathrm{~mm}$ & $\pm 0.2 \mathrm{~mm}$ & $\pm 0.6 \mathrm{~mm}$ \\
\hline Tilt Rot-x & \pm 0.5 arcmin & $\pm 0.5 \operatorname{arcmin}$ & $\pm 0.6 \operatorname{arcmin}$ & $\pm 1.6 \operatorname{arcmin}$ \\
\hline Tilt Rot-y & \pm 0.4 arcmin & \pm 0.4 arcmin & $\pm 0.2 \operatorname{arcmin}$ & \pm 1.0 arcmin \\
\hline Tilt Rot-z & \pm 0.1 arcmin & \pm 0.1 arcmin & \pm 0.2 arcmin & \pm 0.4 arcmin \\
\hline
\end{tabular}

Optical tolerance analysis leads to alignment specifications of LFT (Table 6), which are derived from the polarization angle variation. The gravitation deformation of LFT is estimated to be $\delta x$ of $-14 \mu \mathrm{m}, \delta y$ of $-23 \mu \mathrm{m}$, $\delta z$ of $22 \mu \mathrm{m}$, which are all reasonably small. Then, we can plan the ground verification and calibration without directional constraints due to gravitational effects. According to a scaled model (see Section 8), the alignment can be achieved with careful design and assembly.

The surface roughness of the reflectors are designed to be $2-4 \mu \mathrm{m}$ in $\mathrm{R}_{a}$ on the scale of $10 \mathrm{~mm}$, which reduces infrared radiation, mainly from the Galactic plane. According to the Ruze fomula $\eta_{e}=\exp \left[-\left(\frac{4 \pi \epsilon}{\lambda}\right)^{2}\right]$, infrared radiation more than 5-10 $\mathrm{THz}(30-60 \mu \mathrm{m})$ can be scattered.

The telescope is tightly covered with aluminum and absorbers to reduce the stray light from the inner surface of the $30 \mathrm{~K}$ V-groove (see Figure 1). The absorber, made of plastic and carbon, is adhered to a panel with epoxy, then the panel is fixed to the $5 \mathrm{~K}$ frame. The cryogenic contraction of the absorber and the epoxy will be carefully designed not to deform the frame.
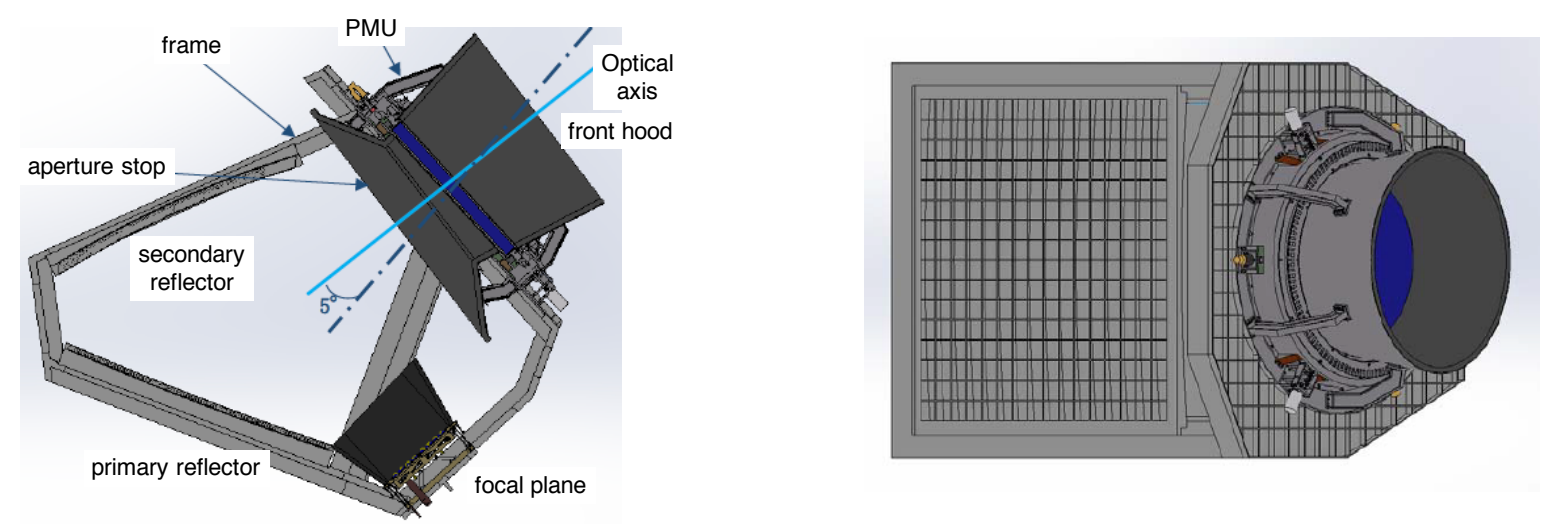

Figure 9. (Left) Lateral view of structural design of LFT. The side panel is covered with millimeter absorbers. (Right) Top view of LFT.

\section{LFT POLARIZATION MODULATION UNIT (PMU)}

A polarization modulation unit with a transmissive sapphire HWP has been developed for LiteBIRD (Figure 10). ${ }^{36-38}$ The progress of the PMU is separately reported. ${ }^{16}$ The PMU/HWP is placed in front of the aperture stop or entrance pupil of $400 \mathrm{~mm}$ diameter. The HWP continuously rotates with $46 \mathrm{rpm}=0.77 \mathrm{~Hz}$. PMU uses superconducting magnets for levitation. ${ }^{36}$ The eddy current and magnetic hysteresis dissipate and increase the temperature of the rotating $\mathrm{HWP}$ from $5 \mathrm{~K}$ to $20 \mathrm{~K}$. The HWP rotation axis is tilted by $5^{\circ}$ with respect to the optical axis to mitigate multiple reflections including optical ghosts between the HWP and the focal plane.

We have derived following the interface specifications on LFT PMU and focal plane from the LFT specifications (Section 3) and system designs during ISAS pre-phase A2. ${ }^{2,3}$

1. The optical effects of the observation frequency of $34-161 \mathrm{GHz}$ due to the PMU are minimized to meet the near and far sidelobes specifications of LFT. 


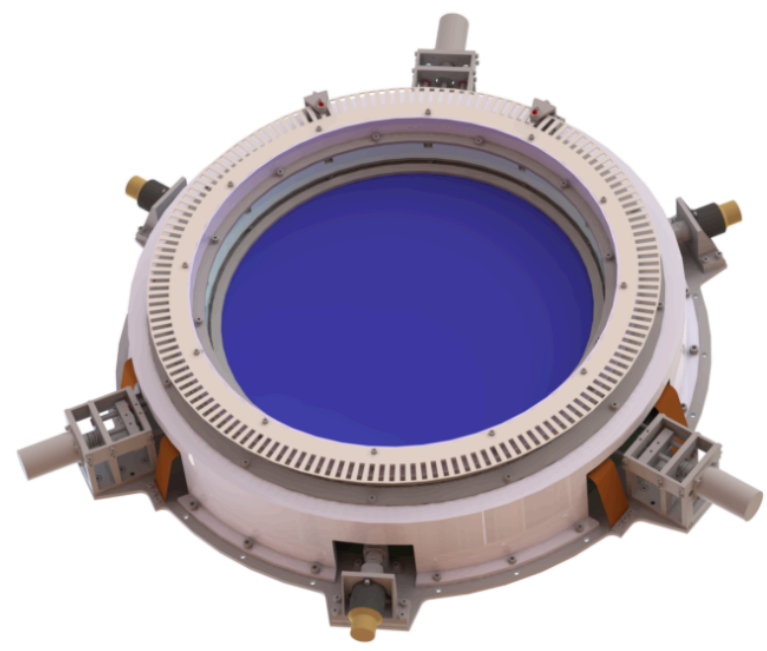

Figure 10. LFT Polarization Modulation Unit (PMU). ${ }^{16}$ The sapphire half-wave plate is shown in blue.

2. The opaque $20 \mathrm{~K}$ parts of PMU are designed to reduce the optical loading.

3. The mass of PMU is $30 \mathrm{~kg}$.

4. The heat loads to the $5 \mathrm{~K}$ stage including the PMU wire harness are less than $3 \mathrm{~mW}$.

5. AC magnetic field variation and DC magnetic field are minimized to reduce the effects on the focal plane.
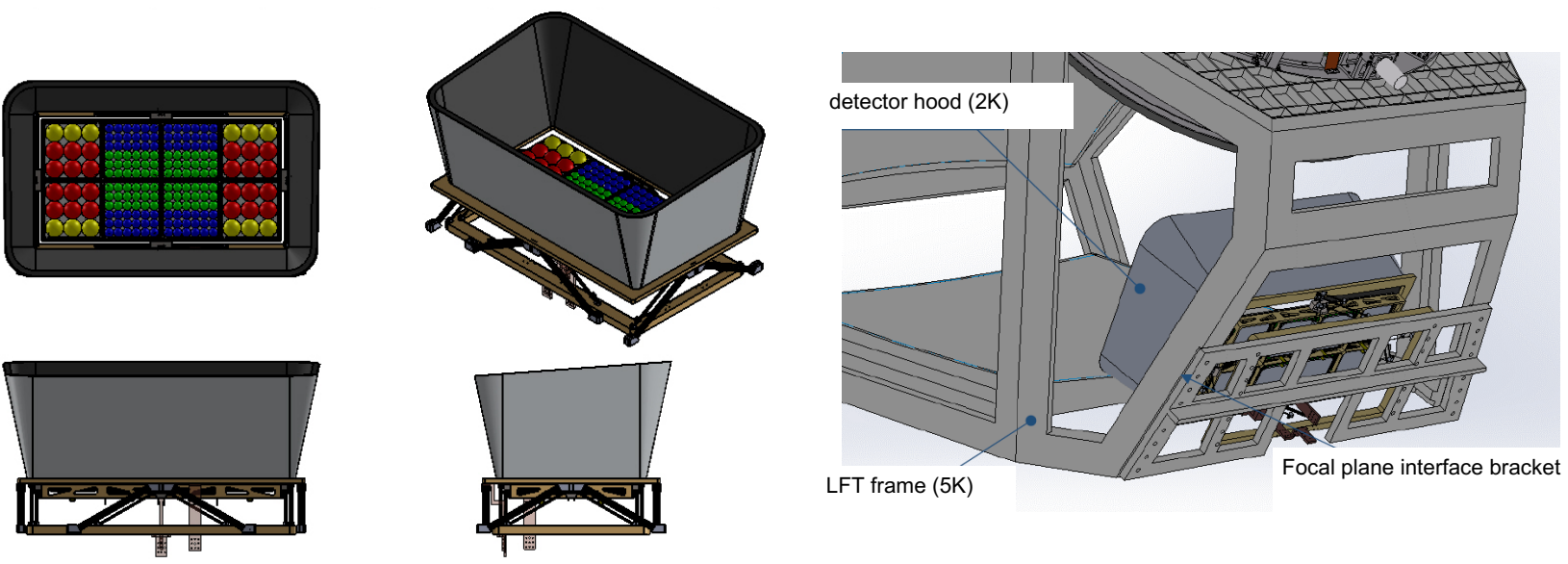

\section{LFT FOCAL PLANE}

Figure 11. (Left) LFT focal plane assembly. (Right) Structural interface between the focal plane and LFT.

The LFT focal plane has been designed and developed with antenna-coupled TES detectors. ${ }^{12}$ The lens and sinuous antenna have broadband capability. ${ }^{31}$ The focal plane with AlMn TES is cooled to $100 \mathrm{mK}$ with ADRs. ${ }^{15}$ The cold readout with SQUID amplifiers is also cooled to $100 \mathrm{mK}$. Cosmic ray mitigation has been extensively investigated. ${ }^{39,40}$ The progress of the LFT focal plane is separately reported. ${ }^{13}$

The focal plane consists of eight square $(10 \mathrm{~cm} \times 10 \mathrm{~cm})$ tiles, as shown in Figure 11. The focal plane is shielded with a hood at $2 \mathrm{~K}$ to reduce stray light (see Figure 2). A quasi-optical metal-mesh low-pass filter ${ }^{17}$ is put in front of square modules to reduce thermal loads from far-infrared radiation of the Galactic plane and the 
$20 \mathrm{~K}$ radiation of PMU. A magnetic shield to reduce magnetic variation from the PMU covers the focal plane except for the optical input. The structural interface at $5 \mathrm{~K}$ between the focal plane and LFT is designed as shown in Figure 11.

The following interface specifications on the focal plane are flown down from the LFT specifications and system designs.

1. The optical efficiency of each detector is higher than 0.69 .

2. The return loss of the feeds in the in-band frequencies is better than $-10 \mathrm{~dB}$.

3. The main beam width of the feeds is consistent with the Gaussian beam radius defined in Table 4 within $5 \%$.

4. The sidelobes of each detector are less than $-17 \mathrm{~dB}$. Figure 7 shows the effects of the feed sidelobes.

5. The optical cross talk among pixels is less than $0.03 \%$.

6. The lower frequency edges of $34 \mathrm{GHz}$ and $60 \mathrm{GHz}$ of the $40 \mathrm{GHz}$ band and the $68 \mathrm{GHz}$ band, respectively, have sharper cut-offs to reduce the contamination of sidelobes of the lower frequencies. Figure 8 shows the beam pattern at $30 \mathrm{GHz}$.

7. The polarization efficiency of the feeds should be higher than $98 \%$, which corresponds to the cross polarization of $<-17 \mathrm{~dB}$.

8. The polarization angle of each detector across the frequency band changes by less than $\pm 5^{\circ}$.

9. The detector noise is basically the photon noise limit of the cosmic microwave background of $2.7 \mathrm{~K}$. The NET is tabulated in Table 1.

10. The common mode $1 / f$ knee noise of the detector module is stable to be better than $100 \mathrm{mHz}$.

11. The $1 / f$ knee of each detector is stable to be better than $20 \mathrm{mHz}$.

12. Micro-vibration of the $5 \mathrm{~K}$ interface is less than $30 \mu \mathrm{G} / \sqrt{\mathrm{Hz}}$ and $80 \mu \mathrm{G} / \sqrt{\mathrm{Hz}}$ over $10-200 \mathrm{~Hz}$ and 200 $500 \mathrm{~Hz}$, respectively. Under this condition, the focal plane shall perform the required sensitivity. This requirement is based on the experience of the Hitomi X-ray satellite. ${ }^{41}$

13. The detector yield including the readout electronics is larger than $80 \%$.

14. The dead time fraction due to cosmic ray glitches is less than 0.05 .

15. The mass of the focal plane assembly is assumed to be $17 \mathrm{~kg}$ without the magnetic shield.

16. The first eigen-frequency of the focal plane is required to larger than $141 \mathrm{~Hz}$ for all three axes.

\section{SCALED MODEL DEMONSTRATION}

A quarter (1/4)-size scaled model of the LFT antenna has been designed and developed to verify the wide-field design. Measured frequencies are also scaled, so the antenna pattern of the scaled model reveals that of the full size.

The near-field measurement system with the scaled LFT has been developed as shown in Figure $12 .{ }^{42}$ Measured amplitude and phase data are transformed to far fields. Figure 12 shows far-field beam patterns at three focal positions (see Figure 6), center, top-right edge, and bottom-right edge, at the frequency of $220 \mathrm{GHz}$, which corresponds to $55 \mathrm{GHz}$ in the full size LFT. We confirmed the suppression of far sidelobes based on the scaled model measurements.

Rotation of polarization angle over the field of view is another key parameter for the wide-field design. A dedicated compact antenna test range (CATR), or a collimated millimeter-wave source has been developed to 

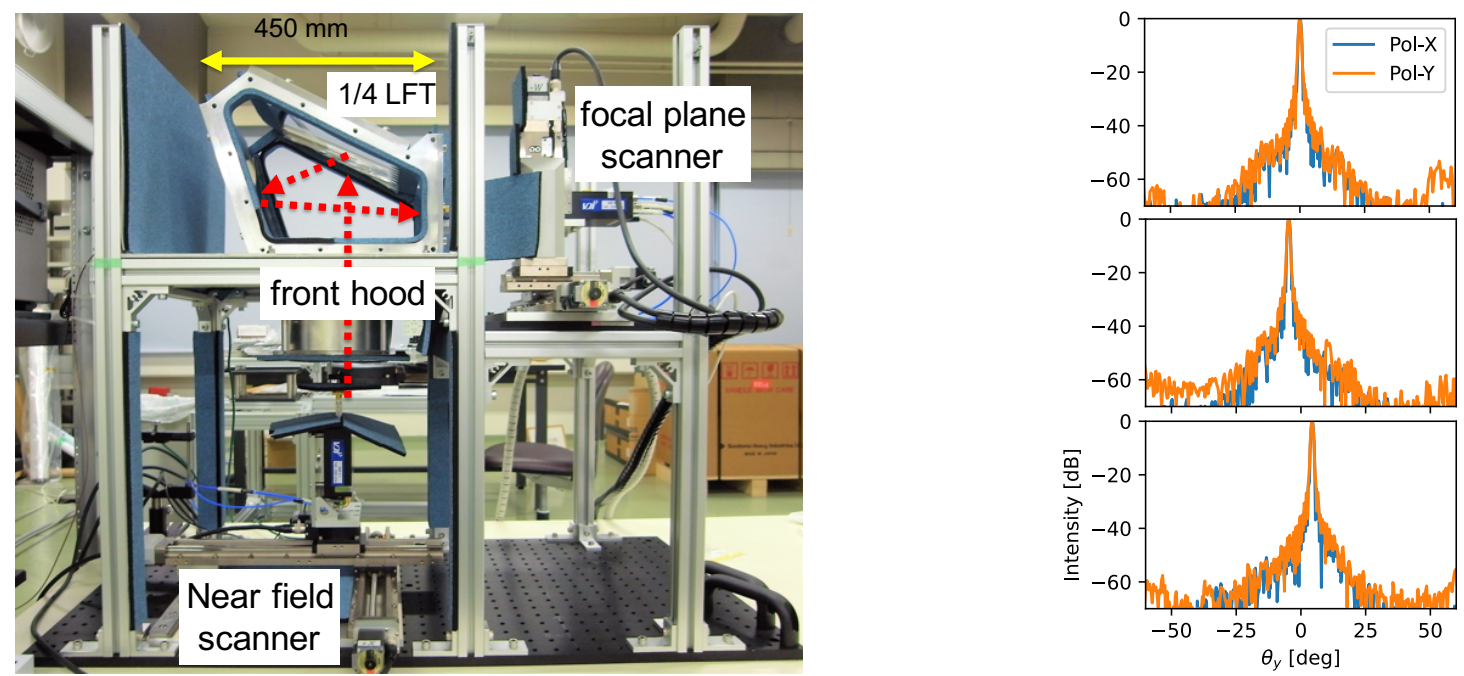

Figure 12. (Left) LFT quarter (1/4) scaled model and the near-field measurement system. ${ }^{42}$ (Right) Far-field patterns of the quarter LFT at the center (top) and edges (middle and bottom) of the focal plane, measured at $220 \mathrm{GHz}$, which corresponds to $55 \mathrm{GHz}$ in the full model. ${ }^{42}$

measure the polarization angle across the wide field of view of the $1 / 4$ LFT. The polarization angle of the $1 / 4$ scaled LFT has been measured with a resolution of $0.1^{\prime} .{ }^{43}$ The polarization angle of polarization $x$ or horizontal polarization was measured to rotate by around $60^{\prime}$ across the focal plane, while the angle of polarization $y$ or vertical polarization rotates by around $30^{\prime}$ across the focal plane.

The structural design of the LFT antenna has been studied with the 1/4-scaled LFT. The frame structure of the 1/4-LFT as shown in Figure 13, was assembled with plates and rectangular bars. The reflector alignment of the assembled 1/4 LFT was measured with a coordinated machine (Mitsutoyo Legex 12128), as shown in Figure 13. The fitted curve of the optical surfaces referring to the aperture center is different from the designed values by $36 \mu \mathrm{m}$ and $22^{\prime \prime}$ at the maximum. The measured alignment met the quarter values of the alignment requirement of Table 6 .
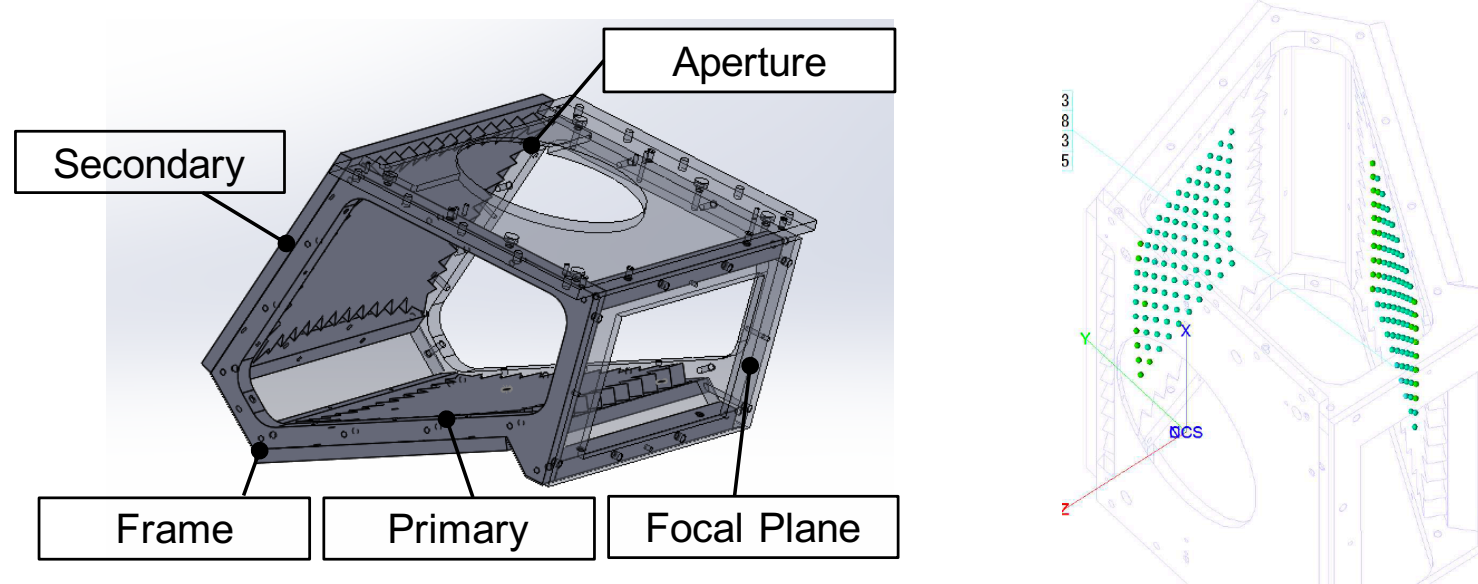

Figure 13. (Left) LFT quarter (1/4) scaled model. (Right) Measurement of reflector surfaces with a coordinated machine. 


\section{VERIFICATION PLAN}

Verification and calibration of a cryogenic telescope at the ground facilities before launch are challenging. A verification plan is tabulated in Table 7 . Two development models (DM/EM and $\mathrm{FM}^{*}$.) are planned. ${ }^{2}$

Table 7. Verification plan of LFT.

\begin{tabular}{|c|c|c|}
\hline & $\mathrm{DM} / \mathrm{EM}$ & FM \\
\hline \multicolumn{3}{|l|}{ LFT-antenna tests at room temperature } \\
\hline Shape measurements with a 3D coordinated machine & $\checkmark$ & $\checkmark$ \\
\hline Millimeter-wave antenna pattern with horns & $\checkmark$ & $\checkmark$ \\
\hline V-grooves/MHFT diffraction & $\checkmark$ & - \\
\hline \multicolumn{3}{|l|}{ LFT-antenna cryogenic tests at $5 \mathrm{~K}$} \\
\hline Strain measurements & $\checkmark$ & - \\
\hline Deformation measurements: photogrammetry or laser sensing & optional & optional \\
\hline Millimeter-wave antenna pattern with horns & optional & optional \\
\hline \multicolumn{3}{|l|}{ LFT AIV and calibration with FP and PMU } \\
\hline Antenna pattern & $\checkmark$ & $\checkmark$ \\
\hline Polarization angle & $\checkmark$ & $\checkmark$ \\
\hline Frequency response & $\checkmark$ & $\checkmark$ \\
\hline
\end{tabular}

The antenna pattern of LFT before integration with the focal plane will be tested at room temperature. A possible method is a near-field beam measurement ${ }^{42}$ or a CATR measurement. ${ }^{43}$

Diffraction effects due to V-grooves and structures of MHFT will be evaluated and modeled to be small enough $(<-60 \mathrm{~dB})$ as designed at room temperature. A structure thermal model (STM) of the mission payload is constructed and tested with mechanical coolers to verify structural and thermal performance. ${ }^{2}$ It will be used to measure the electromagnetic effects of V-grooves at room temperature.

Then, the cryogenic deformation of LFT will be measured to be small enough, as designed. There are a few methods to measure cryogenic deformation of LFT: 1) strain measurements with strain gauges; 2) photogrammetric measurements; and 3) laser reflection measurements.

To verify the requirements of LFT and to calibrate LFT with the focal plane and the PMU, we have a plan to build a beam measurement system in a cryogenic environment. There are three methods to measure cryogenic beam patterns, polarization angles and spectral response (Table 8). One approach is near-field beam measurements in front of the front hood of LFT. To obtain the far-field pattern from the near-field measurements, the phase distribution must be retrieved with a reference source. ${ }^{44}$

Another method is direct measurement of far-field pattern with a collimated source or a compact antenna test range (CATR), which needs larger volume for the cryogenic environment, as shown in Figure 14. This concept has three merits over the phase retrieval near-field beam measurement.

1. The polarization angle of LFT is also measured with a collimated beam, as demonstrated by H. Takakura et al. $2020 .^{43}$

${ }^{*}$ DM: demonstration model, EM: engineering model, FM: flight model

Table 8. Possible cryogenic RF measurements. CATR: compact antenna test range. CW : continuous wave/coherent source.

\begin{tabular}{|l|c|c|c|}
\hline & Near Field & CATR with CW & CATR with blackbody \\
\hline Phase retrieval & necessary & unnecessary & unnecessary \\
\hline Volume & compact & large & large \\
\hline Time & longer & fast & faster \\
\hline Standing wave & no concern & little concern & no concern \\
\hline Pol. angle & difficult & possible & possible \\
\hline Spectral response & difficult & possible & - \\
\hline
\end{tabular}




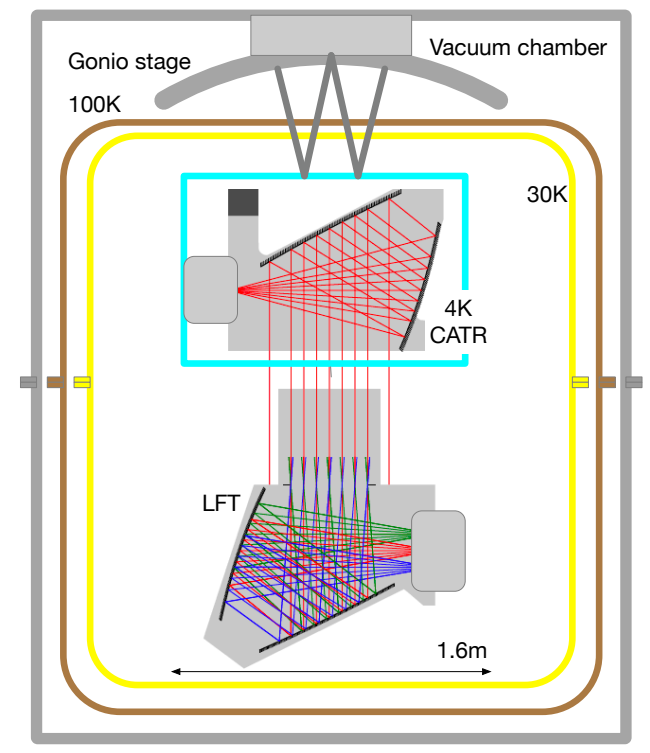

Figure 14. Schematic drawing of cryogenic set-up with a CATR (compact antenna test range), which moves threedimensionally with two Gonio stages. It is planned to measure co-polar and cross-polar beam pattens, polarization angle, and spectral response of LFT with CW and blackbody sources.

2. The frequency spectral response is measured with a broadband coherent source. A few broadband photomixers have been demonstrated at millimeter-wave frequencies. ${ }^{45,46}$

3. It is possible to measure beam patterns with continuum sources as well as coherent sources. Beam measurements with a continuum source are faster than those of multiple frequencies with coherent sources.

In either method, it is crucial to de-couple the mechanics at room temperature from the sources at cryogenic temperature, or to develop moving mechanics operated at low temperature.

\section{SUMMARY}

Based on the performance specifications of LFT, a wide field-of-view design has been studied as well as structural and thermal designs. A 1/4-scaled model of LFT has been developed to verify the design. The measured beam pattern was consistent with the optical model at a level of $-50 \mathrm{~dB}$. Interface specifications of the LFT PMU and LFT focal plane are presented. The verification scheme of LFT is planned as the ISAS/JAXA pre-phase A activity.

\section{ACKNOWLEDGMENTS}

This work is supported in Japan by ISAS/JAXA for Pre-Phase A2 studies, by the acceleration program of JAXA research and development directorate, by the World Premier International Research Center Initiative (WPI) of MEXT, by the JSPS Core-to-Core Program of A. Advanced Research Networks, and by JSPS KAKENHI Grant Numbers JP15H05891, JP17H01115, and JP17H01125. The Italian LiteBIRD phase A contribution is supported by the Italian Space Agency (ASI Grants No. 2020-9-HH.0 and 2016-24-H.1-2018), the National Institute for Nuclear Physics (INFN) and the National Institute for Astrophysics (INAF). The French LiteBIRD phase A contribution is supported by the Centre National d'Etudes Spatiale (CNES), by the Centre National de la Recherche Scientifique (CNRS), and by the Commissariat à l'Energie Atomique (CEA). The Canadian contribution is supported by the Canadian Space Agency. The US contribution is supported by NASA grant no. 80NSSC18K0132. Norwegian participation in LiteBIRD is supported by the Research Council of Norway (Grant No. 263011). The Spanish LiteBIRD phase A contribution is supported by the Spanish Agencia Estatal de Investigación (AEI), project refs. PID2019-110610RB-C21 and AYA2017-84185-P. Funds that support the 
Swedish contributions come from the Swedish National Space Agency (SNSA/Rymdstyrelsen) and the Swedish Research Council (Reg. no. 2019-03959). The German participation in LiteBIRD is supported in part by the Excellence Cluster ORIGINS, which is funded by the Deutsche Forschungsgemeinschaft (DFG, German Research Foundation) under Germany's Excellence Strategy (Grant No. EXC-2094 - 390783311). This research used resources of the Central Computing System owned and operated by the Computing Research Center at KEK, as well as resources of the National Energy Research Scientific Computing Center, a DOE Office of Science User Facility supported by the Office of Science of the U.S. Department of Energy.

\section{REFERENCES}

[1] Hazumi, M. and LiteBIRD collaboration, "LiteBIRD: a small satellite for the study of B-mode polarization and inflation from cosmic background radiation detection," in [SPIE Astronomical Telescopes + Instrumentation], 844219-9 (2012).

[2] Sekimoto, Y. and LiteBIRD collaboration, "Concept design of the LiteBIRD satellite for CMB B-mode polarization," in [Space Telescopes and Instrumentation 2018: Optical, Infrared, and Millimeter Wave], 10698, 106981Y, SPIE (2018).

[3] Hazumi, M. and LiteBIRD Joint Study Group, "LiteBIRD satellite: JAXA's new strategic L-class mission for all-sky surveys of cosmic microwave background polarization," in [Space Telescopes and Instrumentation 2020], SPIE (2020).

[4] Seljak, U. and Zaldarriaga, M., "Signature of gravity waves in the polarization of the microwave background," Phys. Rev. Lett. 78, 2054-2057 (Mar 1997).

[5] Kamionkowski, M., Stebbins, A., Kosowsky, A., and Stebbins, A., "A Probe of Primordial Gravity Waves and Vorticity," Phys. Rev. Lett. 78, 2058-2061 (mar 1997).

[6] Zaldarriaga, M. and Seljak, U., "All-sky analysis of polarization in the microwave background," Phys. Rev. D 55(4), 1830-1840 (1997).

[7] Kamionkowski, M., Kosowsky, A., and Stebbins, A., "Statistics of cosmic microwave background polarization," Phys. Rev. D 55(12), 7368-7388 (1997).

[8] Kamionkowski, M. and Kovetz, E. D., "The Quest for B Modes from Inflationary Gravitational Waves," Annu. Rev. Astron. Astrophys. 54, 227-69 (oct 2016).

[9] Tristram, M., Banday, A. J., Górski, K. M., Keskitalo, R., Lawrence, C. R., Andersen, K. J., Barreiro, R. B., Borrill, J., Eriksen, H. K., Fernandez-Cobos, R., Kisner, T. S., Martínez-González, E., Partridge, B., Scott, D., Svalheim, T. L., Thommesen, H., and Wehus, I. K., "Planck constraints on the tensor-to-scalar ratio," arXiv: 2010.01139v1 (2020).

[10] Montier, L. and LiteBIRD Joint Study Group, "Overview of the Mid- and High-Frequency Telescopes of the LiteBIRD satellite mission," in [Space Telescopes and Instrumentation 2020], SPIE (2020).

[11] Lamagna, L. et al., "The optical design of the Litebird Middle and High Frequency Telescope," in [Space Telescopes and Instrumentation 2020], SPIE (2020).

[12] Suzuki, A. et al., "The LiteBIRD Satellite Mission - Sub-Kelvin Instrument," J. of Low Temperature Physics 193, 1048-1056 (2018).

[13] Westbrook, B. et al., "Detector fabrication development for the LiteBIRD satellite mission," in [Space Telescopes and Instrumentation 2020], SPIE (2020).

[14] Hasebe, T., Sekimoto, Y., Dotani, T., Mitsuda, K., Shinozaki, K., and Yoshida, S., "Optimization of cryogenic architecture for LiteBIRD satellite using radiative cooling," Journal of Astronomical Telescopes, Instruments, and Systems 5(4), 044002 (2019).

[15] Duval, J.-M., Prouvé, T., Shirron, P., Shinozaki, K., Sekimoto, Y., Hasebe, T., Vermeulen, G., André, J., Hasumi, M., Montier, L., and Mot, B., "LiteBIRD Cryogenic Chain: $100 \mathrm{mK}$ Cooling with Mechanical Coolers and ADRs," Journal of Low Temperature Physics 199, 730-736 (2020).

[16] Sakurai, Y. et al., "Breadboard model of polarization modulator unit based on a continuous rotating halfwave plate for low frequency telescope of LiteBIRD space mission," in [Space Telescopes and Instrumentation 2020], SPIE (2020).

[17] Ade, P. A. R., Pisano, G., Tucker, C., and Weaver, S., "A review of metal mesh filters," in [SPIE], 62750U$62750 \mathrm{U}(2006)$. 
[18] Ishino, H. and LiteBIRD collaboration, "LiteBIRD: lite satellite for the study of B-mode polarization and inflation from cosmic microwave background radiation detection," in [Proc. SPIE], 9904, 99040X (2016).

[19] Ishino, H., Nagata, R., and LiteBIRD working group, "Development of LiteBIRD analysis pipeline and systematics evaluation," in [Proceedings of the 16th Space Science Symposium], (2016).

[20] Nagata, R., "Requirement analysis for LiteBIRD optical system," JAXA Supercomputer System Annual Report April 2017-March 2018 JSS2 Inter-University Research (feb 2019).

[21] Lee, A. T. et al., "LiteBIRD an all-sky cosmic microwave background probe of inflation," Bulletin of the American Astronomical Society 7(51), 286 (2019).

[22] Tsuji, M. et al., "Simulating electromagnetic transfer function from the transmission antennae to the sensors vicinity in LiteBIRD," in [Space Telescopes and Instrumentation 2020], SPIE (2020).

[23] Tran, H., Lee, A., Hanany, S., Milligan, M., and Renbarger, T., "Comparison of the crossed and the Gregorian Mizuguchi-Dragone for wide-field millimeter-wave astronomy," Applied Optics 47(2), 103-109 (2008).

[24] Bernacki, B. E., Kelly, J. F., Sheen, D., Hatchell, B., Valdez, P., Tedeschi, J., Hall, T., and McMakin, D., "Wide-field-of-view millimeter-wave telescope design with ultra-low cross-polarization," in $[S P I E], \mathbf{8 3 6 2}$, 836207-836211 (may 2012).

[25] Young, K. et al., "Optical design of PICO: a concept for a space mission to probe inflation and cosmic origins," in [Space Telescopes and Instrumentation 2018: Optical, Infrared, and Millimeter Wave], 10698, 1242 - 1253, SPIE (2018).

[26] Tran, H. et al., "Optical Design of the EPIC-IM Crossed Dragone Telescope," in [SPIE], 7731, 77311R-15 (2010).

[27] Kashima, S., Hazumi, M., Imada, H., Katayama, N., Matsumura, T., Sekimoto, Y., and Sugai, H., "Wide field-of-view crossed Dragone optical system using anamorphic aspherical surfaces," Appl. Opt. 57(15), 4171-4179 (2018).

[28] "TICRA." http://www.ticra.com/software/grasp/.

[29] Imada, H., Dotani, T., Hasebe, T., Hazumi, M., Inatani, J., Ishino, H., Kashima, S., Katayama, N., Kimura, K., Matsumura, T., Nagata, R., Sekimoto, Y., Sugai, H., Suzuki, A., and Utsunomiya, S., "The optical design and physical optics analysis of a cross-Dragonian telescope for LiteBIRD," in [Space Telescopes and Instrumentation 2018: Optical, Infrared, and Millimeter Wave], 10698, 106984K, SPIE (2018).

[30] "HFSS." https://www.ansys.com/products/electronics/ansys-hfss.

[31] Edwards, J. M., O'Brient, R., Lee, A. T., and Rebeiz, G. M., "Dual-Polarized Sinuous Antennas on Extended Hemispherical Silicon Lenses," IEEE Transactions on Antennas and Propagation 60, 4082-4091 (sep 2012).

[32] "TK-RAM." http://www.terahertz.co.uk/.

[33] Saily, J. and Raisanen, A., "Characterization of Submillimeter Wave Absorbers from 200 - 600 GHz," International Journal of Infrared and Millimeter Waves 5, 71 (2004).

[34] "NASA outgassing data." https://outgassing.nasa.gov/.

[35] "NIST Material Properties References." https://trc.nist.gov/cryogenics/materials/references. htm.

[36] Sakurai, Y., Matsumura, T., Iida, T., Kanai, H., Katayama, N., Imada, H., Ohsaki, H., Terao, Y., Shimomura, T., Sugai, H., Kataza, H., Yamamoto, R., and Utsunomiya, S., "Design and thermal characteristics of a $400 \mathrm{~mm}$ diameter levitating rotor in a superconducting magnetic bearing operating below at $10 \mathrm{k}$ for a cmb polarization experiment," IEEE Transactions on Applied Superconductivity 28, 1-4 (June 2018).

[37] Sakurai, Y. et al., "Design and development of a polarization modulator unit based on a continuous rotating half-wave plate for LiteBIRD," in [Proc. SPIE], 10708, 107080E (2018).

[38] Komatsu, K., Matsumura, T., Imada, H., Ishino, H., Katayama, N., and Sakurai, Y., "Demonstration of the broadband half-wave plate using the nine-layer sapphire for the cosmic microwave background polarization experiment," Journal of Astronomical Telescopes, Instruments, and Systems 5, 1 (nov 2019).

[39] Stever, S. et al., "Cosmic ray glitch predictions, physical modelling, and overall effect on the LiteBIRD space mission," JCAP, in preparation (2020).

[40] Tominaga, M. et al., "Simulation of cosmic ray effects in the cmb b-mode polarization observation satellite litebird," in [Space Telescopes and Instrumentation 2020], SPIE (2020). 
[41] Takei, Y., Yasuda, S., Ishimura, K., et al., "Vibration isolation system for cryocoolers of soft x-ray spectrometer on-board ASTRO-H (Hitomi)," Journal of Astronomical Telescopes, Instruments, and Systems 4, 011216 (feb 2018).

[42] Takakura, H., Sekimoto, Y., Inatani, J., Kashima, S., Imada, H., Hasebe, T., Kaga, T., Takeda, Y., and Okada, N., "Far-Sidelobe Antenna Pattern Measurement of LiteBIRD Low Frequency Telescope in 1/4 Scale," IEEE Transactions on Terahertz Science and Technology 9, 598-605 (nov 2019).

[43] Takakura, H., Sekimoto, Y., Inatani, J., Kashima, S., and Sugimoto, M., "Polarization angle measurement of litebird low frequency telescope scaled model," in [Space Telescopes and Instrumentation 2020], SPIE (2020).

[44] Smith, D., Leach, M., Elsdon, M., and Foti, S., "Indirect Holographic Techniques for Determining Antenna Radiation Characteristics and Imaging Aperture Fields," IEEE Antennas and Propagation Magazine 49, 54-67 (feb 2007).

[45] Hirata, A., Nagatsuma, T., Yano, R., Ito, H., Furuta, T., Hirota, Y., Ishibashi, T., Matsuo, H., Ueda, A., Noguchi, T., Sekimoto, Y., Ishiguro, M., and Matsuura, S., "Output power measurement of photonic millimeter-wave and sub-millimeter-wave emitter at 100-800 GHz," Electron Lett 38, 798 (2002).

[46] Kiuchi, H., Kawanishi, T., and Kanno, A., "Wide frequency range optical synthesizer with high-frequency resolution," IEEE Photonics Technology Letters 29, 78-81 (Jan 2017). 